\title{
11. MINERALOGY AND GEOCHEMISTRY OF CRETACEOUS AND CENOZOIC ATLANTIC SEDIMENTS OFF THE IBERIAN PENINSULA (SITE 398, DSDP LEG 47B)
}

\author{
Hervé Chamley, ${ }^{1}$ Pierre Debrabant, ${ }^{2}$ Janine Foulon, ${ }^{2}$ Ghislaine Giroud d'Argoud, ${ }^{1}$ Claude Latouche, ${ }^{3}$ \\ Noéle Maillet, ${ }^{3}$ Henri Maillot, ${ }^{2}$ and Frédéric Sommer ${ }^{4}$
}

\begin{abstract}
Subcontinuously cored from Hauterivian to recently deposited sediments, DSDP Site 398 was studied mineralogically and geochemically by routine and specific investigations. The whole series, lithologically quite diversified, contains minerals and associated chemical elements whose origin is chiefly detrital. The clay minerals in particular are inherited from Iberian soils and/or rocks, including smectite, attapulgite (palygorskite), and sepiolite. One cannot detect any obvious evidence of diagenesis with depth of burial, volcanism, or hydrothermalism. The only in-situ modifications observed are the dissolution of carbonates (changes in the CCD and biogenic supply); possible moderate degradation of smectite in Cretaceous black shales with local growth of CA, Fe, and $\mathrm{Mn}$ carbonates; and the probably formation of zeolites and cristobalite. These data lead to consideration of the mineralogical and chemical elements as markers of the paleoenvironment on the European continent and margin. By our studies nine zones are defined and their significance discussed.

The Cretaceous and Paleogene sediments are characterized by the abundance of smectite rich in $\mathrm{Fe}, \mathrm{Ti}, \mathrm{Cr}, \mathrm{V}$, and light rare earth elements. This mineral originates chiefly from erosion of soils developed in low relief continental zones during an arid, warm climate with contrasts in seasonal humidity. The climate shows numerous fluctuations, with temperature and wetness maxima during the middle Albian and the upper Paleocene-middle Eocene ages. Starting in the Oligocene, an irregular augmentation of primary minerals (illite, chlorite, quartz, feldspars, amphiboles) more or less pedogenically weathered (irregular mixed-layers) reflects cooling by stages, resulting in the Neogene glaciations.

The aperiodic instability of the oceanic margin together with climatic effects, especially during the Albian and Paleocene-Eocene, fostered a supply of detrital minerals to the open sea. These detrital minerals include attapulgite, sepiolite, perhaps clinoptilolite and smectite, accompanied by some $\mathrm{Mg}$ and $\mathrm{Mn}$. These reworked chemical deposits are often mixed with material derived from river basins, whose erosion was favored by the continental margin tectonics. The repercussions of global tectonics on margins are probably also responsible for the temporary supply of primary minerals and kaolinite, especially during Barremina (rifting) and late Aptian (spreading) time.

During the middle Santonian, the sudden arrival of primary minerals and minerals formed in upstream soils (illite, chlorite, sandy silicates, kaolinite), and continuing at least until the early Maestrichtian, could be caused by Pyrenean tectonics s. 1., or by a worldwide regression. This change could also be due to a supply of minerals inherited from high latitudes, by the establishment of a north-south oceanic circulation during a major opening stage of the North Atlantic (separation of Greenland and Canada ?). By middle-upper Oligocene time, the increase of primary minerals together with $\mathrm{Ca}$ and $\mathrm{Sr}$ indicates the establishment of the present-day type of deep oceanic circulations.
\end{abstract}

\footnotetext{
${ }^{1}$ Oceanographic center, Luminy, 13288 Marseille Cx 2, France. Present address: Lille University, 59650 Villeneuve D'Ascq, France.

${ }^{2}$ Lille University, 59650 Villeneuve D'Ascq, France.

${ }^{3}$ Bordeaux University, 33405 Talence, France.

${ }^{4}$ Compagnie Français des Pétroles, Crs Galliéni, 33000 Bordeaux, France.
} 


\section{INTRODUCTION}

Site 398 is located in 3890 meters of water, south of Vigo Seamount $\left(40^{\circ} 57.6^{\prime} \mathrm{N} ; 10^{\circ} 43.1^{\prime} \mathrm{W}\right)$. Of the five holes we drilled $(398,398 \mathrm{~A}, 398 \mathrm{~B}, 398 \mathrm{C}$, and 398D), the last one bottomed in Hauterivian sediments at 1740 meters. Only a few gaps exist near the Hauterivian/ Barremian, Cenomanian/Campanian, and Cretaceous/ Tertiary boundaries. Sampling was continuous in the Cretaceous and Paleocene. Five lithologic units were recognized in the holes (Site Report, this volume) as depicted in Figure 1 (back pocket foldout) and summarized below.

\section{Unit 1, With Three Sub-Units}

Sub-unit 1A (Cores 1 to 4 of Hole 398, 1 and 2 of Hole 398A, and 1 and 3 of Hole 398D; 0 to $326 \mathrm{~m}$ ); marly nannofossil ooze to nannofossil ooze; Pleistocene, Pliocene, upper Miocene.

Sub-unit 1B (Cores 3 to 6 of Hole 398D; 326 to 430 $\mathrm{m})$; marly nannofossil chalk to nannofossil chalk; upper and middle Miocene.

Sub-unit 1C (Cores 7 to 19 of Hole 398D; 430 to 590 $\mathrm{m})$; siliceous marly chalk transitional; lower Miocene, Oligocene.

Unit 2 (Cores 19 to 38 of Hole 398D; 590 to $765 \mathrm{~m}$ ); Siliceous Marly Chalk, Mudstone, Slumps; Oligocene, Eocene, Paleocene

\section{Unit 3, With Two Sub-Units}

Sub-unit 3A (Cores 39 to 44 of Hole 398D; 765 to 832 m); red marly chalk; Paleocene, Maestrichtian.

Sub-unit 3B (Cores 45 to 55 of Hole 398D; 832-945 m); barren mudstone, claystone; Campanian, Santonian.

\section{Unit 4, Including Three Sub-Units}

Sub-unit 4A (Cores 56 to 74 of Hole 398D; 945 to $1183 \mathrm{~m}$ ); dark mudstone to claystone, only slightly dolomitic laminated, occasionally bioturbated; middle and lower Cenomanian, Vraconian, upper and middle Albian.

Sub-unit 4B (Cores 79 to 102 of Hole 398D; 1183 to $1401 \mathrm{~m}$ ); dark shales, laminated to bioturbated, fairly abundant dolomite lenses, beds, laminae, molluskan debris, minor gypsum layers; middle and lower Albian.

Sub-unit 4C (Cores 103 to 130 of Hole 398D; 1401 to $1667 \mathrm{~m})$; thin turbiditic sandstone, siltstone, some mudflows-debris flows, mudchip conglomerate, interbedded with dark shales; Aptian, Barremian.

Unit 5 (Cores 131 to 138 of Hole 398D; 1667 to 1740 m); Nannofossil Limestone, Varved Dark Brown to Gray Muds; Barremian, Upper Hauterivian

The aim of this paper is to provide a geological interpretation of different mineralogical and geochemical studies made in several laboratories. The following analyses were made:

1) Mineralogy by X-ray diffraction of the whole rock and clay fraction of 215 Cenozoic samples (by C. L., N.M.).

2) Mineralogy by X-ray diffraction of the whole rock and clay fraction of 230 Cretaceous samples (by F.S.).

3) Mineralogy (H.C., G.G.A.) and geochemistry (P.D., J.F., H.M.) on 89 selected samples covering the entire interval drilled.

\section{METHODS}

\section{X-Ray Methods}

\section{Cretaceous Sediments}

Bulk mineralogy: The rock was dried at $70^{\circ} \mathrm{C}$ and crushed to approximately $40 \mu \mathrm{m}$. The method of obtaining semiquantitative determinations was by estimating the absorption coefficient of each sample and then comparing its diagram with patterns of external standards. The diffractometer was a Philips 1130 and operating conditions were as follows: nickel-filtered copper $\mathrm{k} \alpha$ radiation at $40 \mathrm{kV}, 20 \mathrm{~mA}$; back monochromator, scanning speed $2^{\circ} 2 \theta$ per minute.

Clay mineralogy: The samples were mixed with distilled water for 10 hours, then decarbonated in $10 \mathrm{~N}$ hydrochloric acid. The excess acid was removed by successive centrifugations. The $<2 \mu \mathrm{m}$ fraction was collected by decantation using Stokes Law, then oriented aggregates were made on glass slides. A Philips X-ray diffractometer with copper Ni-filtered radiation was used to run three scans as follows: (a) from $2^{\circ}$ to $22^{\circ} 2 \theta$ $\left(8^{\circ} / \mathrm{min}\right)$ on natural sample; (b) from $2^{\circ}$ to $22^{\circ} 2 \theta$ $\left(8^{\circ} / \mathrm{min}\right)$ on glycolated sample; and (c) from 20 to $26^{\circ}$ $2 \theta\left(2^{\circ} / \mathrm{min}\right)$ on natural sample.

Quantitative determinations were carried out using peak heights above the background; the amount of each mineral was considered to be directly proportional to the peak height. The $10 \AA$ peak (natural sample) was used for illite and the $18 \AA$ peak (glycolated sample) for smectite. The amount of irregular mixed-layer (illitesmectite) was estimated by difference in diagram traces between "natural" and "glycol" samples, between $11 \AA$ and $13 \AA$. The $7 \AA$ peak gave the total kaolinite + chlorite; the ratio of these two minerals being deduced from peak height ratios at $3.58 \AA$ and $3.54 \AA$, respectively. The percentage total was summed to 100 .

\section{Tertiary Sediments}

Bulk mineralogy: Dried and pulverized samples of total sediments were analyzed according to the powder diagrams method using an internal standard (corindon). By comparison with synthetic reference samples, this method resulted in semiquantitative estimates of quartz, calcite, dolomite, and feldspars (alkali and plagioclase). 
The diffractometer was a Philips 1310 and operating conditions were as follows: nickel-filtered copper $k \alpha$ radiation at $40 \mathrm{kV}, 20 \mathrm{~mA}$; automatic sample changer; step-scanning device and monitoring system of the goniometer.

Clay mineralogy: The entire sediment sample was dispersed in pure water using mechanical agitation. Samples with high carbonate content were first treated with $10 N \mathrm{HCl}$.

After two or three washings in pure water, the $<2 \mu \mathrm{m}$ subfractions were separated by gravity settling. After centrifugation of the $<2 \mu \mathrm{m}$ fraction, the thick paste that resulted was spread across two slides with a standard laboratory spatula. The amount of clay per $\mathrm{cm}^{2}$ was controlled by the amount transferred to the slide. The first slide was saturated with ethylene glycol before analysis. The second slide was scanned untreated, then heated at $550^{\circ} \mathrm{C}$ for one hour before being analyzed.

The diffractometer was a CGR Theta 60 with operating conditions as follows: $\mathrm{Cu} k \alpha$ radiation (very thin focus, $0.1 \times 5 \mathrm{~mm}$ ) selected with a Guinier monochromator: $40 \mathrm{Kv}, 10 \mathrm{~mA}$; scanning speed: $0.5^{\circ} \mathrm{\theta} / \mathrm{min}$. The identification of minerals was made from typical reactions of minerals with classical treatment (Brown, 1961; Thorez, 1975). Semiquantitative estimations of the different minerals were made from diagrams of the glycolated slides. The height of 001 peaks was used to determine the percentage of smectite $(17 \AA)$, illite $(10 \AA)$, and kaolinite + chlorite $(7.1 \AA)$, a distinction between chlorite and kaolinite being made on the basis of the difference between the 002 reflection of kaolinite and the 004 reflection of chlorite.

\section{Selected Samples}

The sample preparation was similar to that above. Both oriented aggregates and oriented pastes were made from the $<2 \mu \mathrm{m}$ fraction. A CGR Theta 60 diffractometer (copper radiation focused by a quartz curvedcrystal monochromator) was used to run the X-ray diffraction scans at $1^{\circ} 2 \theta / \mathrm{min}$. A receiving slit of $1.25 \mathrm{~mm}$ was used for a better determination of mixed-layer minerals. Beside the ethylene-glycol treatment (expansion of smectites), a hydrazine-hydrate treatment was used to characterize the kaolin minerals. The heat treatment consisted of two hours at $490^{\circ} \mathrm{C}$. Semiquantitative evaluations were based on the peak heights and areas; smectite and attapulgite were corrected in addition to peak height, and well-crystallized kaolinite was reduced with regard to middle crystallized illite or chlorite. The balance between chlorite and kaolinite was made from peak height ratios (respectively, $3.54 \AA$ and $3.58 \AA$ ). When this ratio was $1: 1$, the amount of chlorite was assumed to be twice that of kaolinite. Additional data were provided by electron-microscope observations.

Quantitative data show differences between routine studies and studies on selected samples, because the former entail peak height of determinations, whereas the latter are based on heights with correction factors. However, reflective variations of the minerals are paral- lel in both types of studies, which facilitates a combined description of the results and comments.

\section{CHEMICAL ANALYSIS ON SELECTED SAMPLES}

\section{Technique}

The samples were dried at $105^{\circ} \mathrm{C}$, then ground and homogenized. Sample portions $(0.2 \mathrm{~g})$ were subjected to alkaline fusion, then solubilized by $\mathrm{HCl}$ and diluted to $100 \mathrm{ml}$. This treatment allowed the gravimetric determination of $\mathrm{SiO}_{2}$, colorimetric determination of $\mathrm{P}_{2} \mathrm{O}_{5}$, and spectrophotometric determination of $\mathrm{Fe}, \mathrm{CaO}$, $\mathrm{MgO}$, and $\mathrm{Al}_{2} \mathrm{O}_{3}$ (by atomic absorption). Then 2-gram samples were submitted to fluroperchloric treatment, then solubilized by $\mathrm{HCl}$ and diluted to $100 \mathrm{ml}$. That dilution was used for the colorimetric analysis of $\mathrm{TiO}_{2}$, the spectrophotometric analysis of $\mathrm{Na}_{2} \mathrm{O}$ and $\mathrm{K}_{2} \mathrm{O}$ (by emission) and also for traces of $\mathrm{Mn}, \mathrm{Zn}, \mathrm{Li}, \mathrm{Ni}, \mathrm{Cr}, \mathrm{Sr}$, $\mathrm{Co}, \mathrm{Cu}, \mathrm{Pb}$, and $\mathrm{V}$ (by atomic absorption).

The apparatus used for emission and atomic absorption is a type 503 Perkin-Elmer spectrophotometer which was used following the methods of Pinta (1971): base solution (for major elements) or complex synthetic solution (for trace elements) to which 5 per cent of lanthane in chlorhydric solution was added.

\section{Statistical Study}

The large number of analytical data allows a search for the dependencies and relationships between them. The statistical aspects are defined by the correlation coefficient between pairs of chemical elements; the significance of these coefficients sometimes was reinforced by a Fisher test. That technique provides confidence limits in which the correlation coefficient occurs with a probability of 0.95 .

The data can be searched if selected variables (factors) do not have a privileged role in the whole set of variables. Consider an abstract space (E) of $m$ dimensions, number of variables (e.g., $m=18$ ) brought back to a reference in which each axis corresponds to a variable. $N$ samples are available (e.g., $n=18$ ), each with $\mathrm{m}$ measurements which can be associated with a vector $(\mathrm{E})$, the component of which on the $\mathrm{SiO}_{2}$ axis is the measurement of the $\mathrm{SiO}_{2}$ variable in that sample. The extremities of those vectors form a cloud of points which can present accumulations in certain privileged directions, named common factors.

In the factorial analysis in the $\mathrm{R}$ mode, only the search for factors in orthonormal reference was carried out, using an iterative method on the correlation matrix (estimation of the communities) and utilizing diaganolization of a square, real symmetrical matrix by iterative calculation of proper values of decreasing module.

The same process was repeated starting from three factors and increasing the number of common factors by one unit each time and estimating the percentage of variation. The initial cloud is represented in a subspace of (E) dimension $r<m, r$ being the number of factors (e.g., $r=5$ ); thus, only 60 to 90 per cent of the binding 
is represented. The components of a common factor of the initial variables provide correlations, the comparisons of which allows the groups of variables of greatest influence to be traced thus defining the geological origin of the factor.

\section{RESULTS}

The geochemical and mineralogical results permit the determination of 9 zones (Figures 1 [back pocket foldout] and 2, Table 1).

\section{Zone 9: Cores 398D-138 through 131; Hauterivian, Lower Barremian}

Zone 9 consists of lithologic Unit 5 and is characterized by the abundance of calcite in the whole rock and well-crystallized smectite in the clay fraction. Illite, traces of kaolinite and (sometimes) chlorite, and mixedlayers of illite-smectite and illite-vermiculite are also present. Locally, the electron microscope shows the presence of short and rare fibers related to traces of attapulgite or sepiolite (Plate 1, Figure 1). Quartz and feldspars are rare. Ca content is greater than at any other level of the site. Trace elements are characterized
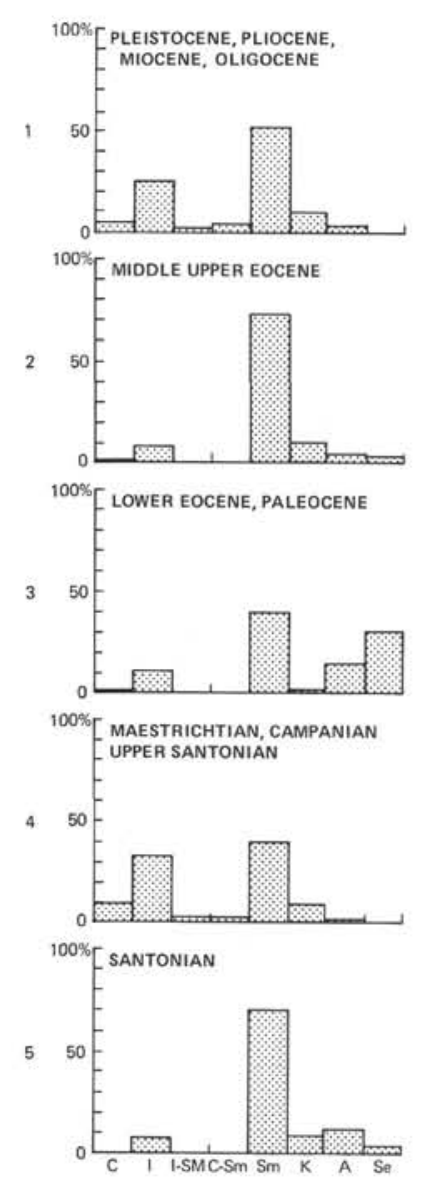
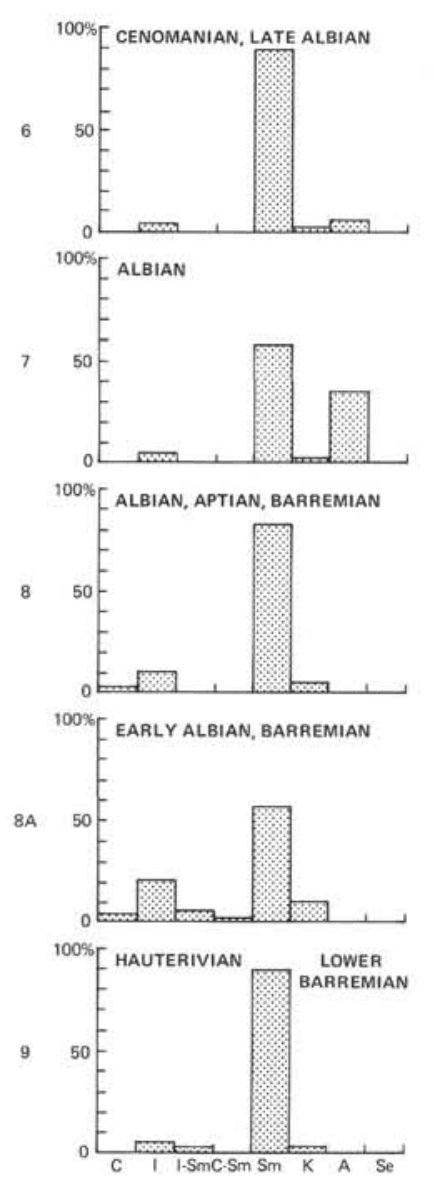

Figure 2. DSDP Site 398. Mineralogical zonation after clay data (quantitative estimations by $\mathrm{H}$. Chamley). $\mathrm{C}=$ chlorite, $I=$ illite, $I$-Sm $=$ illite-smectite mixed-layers, $C$-Sm $=$ chlorite-smectite mixed-layers, $\mathrm{Sm}=$ smectite, $K=$ kaolinite, $A=$ attapulgite (palygorskite), $\mathrm{Se}=$ sepiolite. by a relatively low amount of $\mathrm{Sr}$, the percentage of which is less than those measured in the overlying sediments $\left(10^{3} \mathrm{Sr} / \mathrm{CaO}=0.6\right)$.

\section{Zone 8: Cores 398D-130 through 79; Barremian, Aptian, Lower and Middle Albian}

The clay fraction of sediments in Zone 8 is still dominated by smectite, but kaolinite (as well as quartz and feldspars in the whole rock) increase above the Hauterivian/Barremian boundary. Concurrently, the $\mathrm{Al}_{2} \mathrm{O}_{3} / \mathrm{Fe}$ ratio increases and the $\mathrm{MgO} / \mathrm{K}_{2} \mathrm{O}$ ratio decreases. Statistical studies show that within this zone, the variation of numerous elements $(\mathrm{Mg}, \mathrm{Na}, \mathrm{Cu}, \mathrm{Co}$, $\mathrm{Pb}, \mathrm{Fe}$, as well as $\mathrm{Ca}$ and $\mathrm{Sr}$ ) is independent of that of other variables. This fact can be related to the numerous variations encountered in lithological Sub-units $4 \mathrm{C}$ and 4B which constitute the zone. The only significant correlation is that of $\mathrm{Zn}$ with silicates. Also noteworthy is that the highest phosphatic content at Site 398 is in Sample 398D-93-6, $12 \mathrm{~cm}$.

Quartz and feldspars associated with pyrite are abundant near the bottom of this zone; calcite is present and exhibits large-scale variations until it disappears above 1400 meters, at the Sub-unit 4C/4B boundary. Siderite and/or rhodocrosite are abundant in certain levels (i.e., Samples 398D-118-6, $84 \mathrm{~cm}$ and 398D-98-2, $34 \mathrm{~cm}$ ). These minerals are localized at the contact of black reduced levels; gypsum crystals are also sometimes present (i.e., Sample 398D-93-6, $100 \mathrm{~cm}$ ).

In the clay fraction, large amounts of illite and irregular mixed-layers (illite-smectite, illite-vermiculite, and chlorite-smectite) occur at levels where smectite is less abundant and badly crystallized and where organic carbon reaches the highest values found at this Site (Site Report, this volume; unpublished data on our Zone 8A samples, B. Jouglet, Lille, France). Palygorskites are absent (Plate 1, Figure 2) or exceptional (Sample 398D-101-4, $38 \mathrm{~cm}$ ). Zeolites are present at several levels and are sometimes markedly abundant (Sample 398D-117-4, 52-53 cm). ${ }^{5}$

Clinoptilolite is associated with cristobalite, quartz, plagioclases, and tourmaline in micaceous siltstones interbedded with bioclastic and organic intervals where radiolarians and siliceous sponge spicules are present.

\section{Zone 7: Cores 398D-78 through 73; Middle Albian}

Zone 7 includes the upper part of Sub-unit 4B and the lower part of Sub-unit $4 \mathrm{~A}$, and is characterized by the first occurrence of abundant palygorskite (attapulgite). Fibers are short, lath-shaped, electron translucent, and independent of other minerals (Plate 1, Figures 3 and 4). Kaolinite and illite decrease, while chlorite and mixedlayer clays almost disappear. The bulk mineralogy shows the reappearance of calcite and a decrease of quartz. Geochemistry also shows several changes: (1) a

${ }^{5}$ G. S. Odin, Paris, also determined zeolites in Samples 398D-119$6,30 \mathrm{~cm} ; 398 \mathrm{D}-118-2,72 \mathrm{~cm}$; and 398D-116-3, $80 \mathrm{~cm}$ (personal communication). 
TABLE

Detailed Results of Geochemical Analyses, DSDP Site 398

\begin{tabular}{|c|c|c|c|c|c|c|c|c|c|c|c|c|c|c|c|c|c|c|c|}
\hline $\begin{array}{c}\text { Sample } \\
\text { (Interval in cm) }\end{array}$ & $\begin{array}{l}\mathrm{SiO}_{2} \\
(\%)^{2}\end{array}$ & $\underset{(\%)}{\mathrm{Al}_{2} \mathrm{O}_{3}}$ & $\begin{array}{l}\mathrm{CaO} \\
(\%)\end{array}$ & $\begin{array}{l}\mathrm{MgO} \\
(\%)\end{array}$ & $\begin{array}{l}\mathrm{Na}_{2} \mathrm{O} \\
(\%)\end{array}$ & $\begin{array}{l}\mathrm{K}_{2} \mathrm{O} \\
(\%)\end{array}$ & $\underset{(\%)}{\mathrm{TiO}_{2}}$ & $\mathrm{P}_{2} \mathrm{O}_{5}$ & $\begin{array}{l}\mathrm{Fe} \\
(\%)\end{array}$ & $\begin{array}{c}\mathrm{Mn} \\
(\mathrm{ppm})\end{array}$ & $\underset{(\mathrm{ppm})}{\mathrm{Zn}}$ & $\underset{(\mathrm{ppm})}{\mathrm{Li}}$ & $\underset{(\mathrm{ppm})}{\mathrm{Ni}}$ & $\underset{(\mathrm{ppm})}{\mathrm{Cr}}$ & $\begin{array}{c}\mathrm{Sr} \\
(\mathrm{ppm})\end{array}$ & $\begin{array}{c}\mathrm{Co} \\
(\mathrm{ppm})\end{array}$ & $\underset{(\mathrm{ppm})}{\mathrm{Cu}}$ & $\begin{array}{c}\mathrm{Pb} \\
(\mathrm{ppm})\end{array}$ & $\underset{(\mathrm{ppm})}{\mathrm{V}}$ \\
\hline $\begin{array}{l}\text { Hole } 398 \mathrm{~A} \\
2-2,81\end{array}$ & 11.00 & 4.42 & 40.60 & 0.99 & 0.89 & 0.81 & 0.15 & n.d. & 1.08 & 330 & 80 & 14 & 25 & 53 & 1490 & 11 & 24 & 36 & 26 \\
\hline $\begin{array}{l}\text { Hole } 398 \mathrm{~B} \\
1-3,74\end{array}$ & 8.90 & 3.54 & 41.63 & 0.99 & 0.94 & 0.68 & 0.13 & n.d. & 0.85 & 360 & 96 & 11 & 20 & 49 & 1520 & 8 & 32 & 34 & 21 \\
\hline $\begin{array}{l}\text { Hole 398D } \\
2-2,108 \\
3-1,110 \\
4-4,64 \\
6-1,57 \\
8-2,60\end{array}$ & $\begin{array}{r}10.15 \\
12.50 \\
7.95 \\
7.00 \\
16.60\end{array}$ & $\begin{array}{l}3.83 \\
5.10 \\
1.67 \\
2.16 \\
5.60\end{array}$ & $\begin{array}{l}40.58 \\
38.83 \\
47.22 \\
45.30 \\
36.03\end{array}$ & $\begin{array}{l}1.04 \\
0.99 \\
0.66 \\
0.70 \\
1.33\end{array}$ & $\begin{array}{l}0.94 \\
0.74 \\
0.66 \\
0.58 \\
0.69\end{array}$ & $\begin{array}{l}0.78 \\
0.98 \\
0.36 \\
0.50 \\
1.09\end{array}$ & $\begin{array}{l}0.14 \\
0.17 \\
0.07 \\
0.09 \\
0.19\end{array}$ & $\begin{array}{l}\text { n.d. } \\
\text { n.d. } \\
\text { n.d. } \\
\text { n.d. } \\
\text { n.d. }\end{array}$ & $\begin{array}{l}1.05 \\
1.11 \\
0.64 \\
0.58 \\
1.50\end{array}$ & $\begin{array}{l}350 \\
450 \\
260 \\
400 \\
520\end{array}$ & $\begin{array}{l}62 \\
80 \\
53 \\
58 \\
92\end{array}$ & $\begin{array}{r}13 \\
17 \\
6 \\
8 \\
18\end{array}$ & $\begin{array}{l}23 \\
23 \\
16 \\
18 \\
29\end{array}$ & $\begin{array}{l}54 \\
60 \\
41 \\
43 \\
62\end{array}$ & $\begin{array}{l}1415 \\
1465 \\
1740 \\
1850 \\
1200\end{array}$ & $\begin{array}{r}9 \\
10 \\
6 \\
8 \\
11\end{array}$ & $\begin{array}{r}31 \\
15 \\
6 \\
8 \\
8 \\
18\end{array}$ & $\begin{array}{l}34 \\
33 \\
36 \\
35 \\
30\end{array}$ & $\begin{array}{r}32 \\
32 \\
<5 \\
24 \\
39\end{array}$ \\
\hline $\begin{array}{l}12-2,106 \\
15-2,95 \\
20-2,100 \\
23-1,51 \\
26-1,78\end{array}$ & $\begin{array}{l}34.70 \\
14.50 \\
43.55 \\
37.80 \\
45.60\end{array}$ & $\begin{array}{r}10.22 \\
4.91 \\
15.33 \\
10.12 \\
15.03\end{array}$ & $\begin{array}{r}20.99 \\
38.83 \\
11.19 \\
17.14 \\
8.05\end{array}$ & $\begin{array}{l}1.66 \\
0.99 \\
2.49 \\
2.49 \\
3.15\end{array}$ & $\begin{array}{l}0.99 \\
0.50 \\
1.38 \\
1.13 \\
1.31\end{array}$ & $\begin{array}{l}2.08 \\
0.93 \\
2.83 \\
1.68 \\
2.35\end{array}$ & $\begin{array}{l}0.38 \\
0.15 \\
0.49 \\
0.33 \\
0.50\end{array}$ & $\begin{array}{l}\text { n.d. } \\
\text { n.d. } \\
\text { n.d. } \\
\text { n.d. } \\
\text { n.d. }\end{array}$ & $\begin{array}{l}2.38 \\
1.13 \\
3.88 \\
3.03 \\
4.05\end{array}$ & $\begin{array}{r}670 \\
910 \\
2200 \\
3025 \\
1570\end{array}$ & $\begin{array}{r}96 \\
68 \\
151 \\
186 \\
158\end{array}$ & $\begin{array}{l}29 \\
15 \\
51 \\
36 \\
41\end{array}$ & $\begin{array}{l}25 \\
25 \\
64 \\
41 \\
56\end{array}$ & $\begin{array}{r}78 \\
59 \\
91 \\
104 \\
115\end{array}$ & $\begin{array}{r}950 \\
1375 \\
512 \\
700 \\
390\end{array}$ & $\begin{array}{r}12 \\
8 \\
18 \\
14 \\
23\end{array}$ & $\begin{array}{r}8 \\
12 \\
27 \\
15 \\
24\end{array}$ & $\begin{array}{l}20 \\
34 \\
25 \\
27 \\
28\end{array}$ & $\begin{array}{l}53 \\
29 \\
68 \\
68 \\
81\end{array}$ \\
\hline $\begin{array}{l}27-1,30 \\
28-1,39 \\
29-1,1 \\
29-1,82 \\
30-2,17\end{array}$ & $\begin{array}{l}45.85 \\
42.35 \\
26.15 \\
34.10 \\
42.80\end{array}$ & $\begin{array}{r}15.03 \\
14.25 \\
8.10 \\
10.30 \\
11.30\end{array}$ & $\begin{array}{r}9.62 \\
12.77 \\
27.63 \\
17.70 \\
12.77\end{array}$ & $\begin{array}{l}3.11 \\
3.07 \\
2.24 \\
2.16 \\
2.57\end{array}$ & $\begin{array}{l}1.20 \\
1.25 \\
0.85 \\
1.05 \\
1.24\end{array}$ & $\begin{array}{l}2.60 \\
2.28 \\
1.65 \\
2.10 \\
2.44\end{array}$ & $\begin{array}{l}0.53 \\
0.44 \\
0.28 \\
0.35 \\
0.44\end{array}$ & $\begin{array}{l}\text { n.d. } \\
\text { n.d. } \\
\text { n.d. } \\
\text { n.d. } \\
\text { n.d. }\end{array}$ & $\begin{array}{l}3.58 \\
3.18 \\
1.60 \\
2.53 \\
3.25\end{array}$ & $\begin{array}{r}1240 \\
1250 \\
1590 \\
1165 \\
900\end{array}$ & $\begin{array}{l}139 \\
122 \\
142 \\
250 \\
123\end{array}$ & $\begin{array}{l}40 \\
38 \\
21 \\
33 \\
37\end{array}$ & $\begin{array}{l}42 \\
30 \\
23 \\
30 \\
23\end{array}$ & $\begin{array}{r}109 \\
105 \\
74 \\
69 \\
100\end{array}$ & $\begin{array}{l}369 \\
432 \\
740 \\
700 \\
420\end{array}$ & $\begin{array}{r}17 \\
8 \\
8 \\
8 \\
9\end{array}$ & $\begin{array}{l}14 \\
17 \\
35 \\
39 \\
46\end{array}$ & $\begin{array}{l}31 \\
23 \\
29 \\
14 \\
19\end{array}$ & $\begin{array}{l}79 \\
74 \\
47 \\
42 \\
79\end{array}$ \\
\hline $\begin{array}{l}31-2,55 \\
34-1,38 \\
37-1,112 \\
37-1,23 \\
38-1,40\end{array}$ & $\begin{array}{l}44.80 \\
11.15 \\
52.50 \\
30.60 \\
55.80\end{array}$ & $\begin{array}{r}15.87 \\
2.93 \\
15.87 \\
5.38 \\
16.72\end{array}$ & $\begin{array}{r}11.61 \\
42.50 \\
4.13 \\
28.58 \\
3.11\end{array}$ & $\begin{array}{l}2.82 \\
1.95 \\
4.97 \\
2.07 \\
3.73\end{array}$ & $\begin{array}{l}1.25 \\
0.45 \\
1.70 \\
0.95 \\
1.85\end{array}$ & $\begin{array}{l}2.85 \\
0.45 \\
3.10 \\
1.05 \\
2.65\end{array}$ & $\begin{array}{l}0.42 \\
0.11 \\
0.43 \\
0.17 \\
0.48\end{array}$ & $\begin{array}{l}\text { n.d. } \\
\text { n.d. } \\
\text { n.d. } \\
\text { n.d. } \\
0.23\end{array}$ & $\begin{array}{l}3.90 \\
0.80 \\
3.73 \\
1.25 \\
4.05\end{array}$ & $\begin{array}{l}1120 \\
1440 \\
1610 \\
4700 \\
2300\end{array}$ & $\begin{array}{r}118 \\
55 \\
130 \\
140 \\
140\end{array}$ & $\begin{array}{l}43 \\
14 \\
41 \\
16 \\
47\end{array}$ & $\begin{array}{r}38 \\
28 \\
88 \\
32 \\
110\end{array}$ & $\begin{array}{r}100 \\
26 \\
124 \\
32 \\
90\end{array}$ & $\begin{array}{l}400 \\
780 \\
200 \\
380 \\
300\end{array}$ & $\begin{array}{l}12 \\
11 \\
27 \\
10 \\
29\end{array}$ & $\begin{array}{l}31 \\
26 \\
30 \\
24 \\
68\end{array}$ & $\begin{array}{l}32 \\
40 \\
37 \\
39 \\
29\end{array}$ & $\begin{array}{r}84 \\
32 \\
68 \\
32 \\
100\end{array}$ \\
\hline $\begin{array}{l}39-1,32 \\
40-2,51 \\
41-1,125 \\
44-1,5 \\
45-4,58\end{array}$ & $\begin{array}{l}37.55 \\
25.95 \\
31.75 \\
26.85 \\
42.65\end{array}$ & $\begin{array}{l}13.89 \\
10.11 \\
11.62 \\
10.86 \\
16.53\end{array}$ & $\begin{array}{l}17.04 \\
26.51 \\
23.16 \\
27.84 \\
12.59\end{array}$ & $\begin{array}{l}2.98 \\
2.07 \\
1.95 \\
1.87 \\
2.28\end{array}$ & $\begin{array}{l}1.05 \\
0.75 \\
1.00 \\
0.80 \\
1.20\end{array}$ & $\begin{array}{l}1.38 \\
1.28 \\
1.90 \\
1.35 \\
2.85\end{array}$ & $\begin{array}{l}0.40 \\
0.35 \\
0.36 \\
0.43 \\
0.56\end{array}$ & $\begin{array}{l}\text { n.d. } \\
0.23 \\
\text { n.d. } \\
\text { n.d. } \\
\text { n.d. }\end{array}$ & $\begin{array}{l}3.10 \\
2.70 \\
2.60 \\
3.25 \\
4.25\end{array}$ & $\begin{array}{l}1450 \\
1850 \\
1340 \\
1130 \\
1200\end{array}$ & $\begin{array}{l}130 \\
226 \\
105 \\
115 \\
110\end{array}$ & $\begin{array}{l}47 \\
30 \\
33 \\
27 \\
53\end{array}$ & $\begin{array}{l}57 \\
61 \\
39 \\
45 \\
53\end{array}$ & $\begin{array}{r}170 \\
53 \\
106 \\
55 \\
145\end{array}$ & $\begin{array}{l}440 \\
375 \\
369 \\
525 \\
306\end{array}$ & $\begin{array}{l}20 \\
18 \\
11 \\
20 \\
24\end{array}$ & $\begin{array}{l}39 \\
38 \\
35 \\
23 \\
21\end{array}$ & $\begin{array}{l}63 \\
36 \\
46 \\
33 \\
36\end{array}$ & $\begin{array}{r}150 \\
68 \\
74 \\
84 \\
123\end{array}$ \\
\hline $\begin{array}{l}46-1,118 \\
47-2,70 \\
48-2,66 \\
51-2,37 \\
54-2,28\end{array}$ & $\begin{array}{l}38.05 \\
42.50 \\
40.90 \\
52.70 \\
55.70\end{array}$ & $\begin{array}{l}13.98 \\
16.34 \\
15.30 \\
20.41 \\
18.89\end{array}$ & $\begin{array}{r}17.77 \\
12.31 \\
13.57 \\
0.73 \\
0.65\end{array}$ & $\begin{array}{l}2.07 \\
2.32 \\
2.32 \\
3.03 \\
3.73\end{array}$ & $\begin{array}{l}1.15 \\
1.20 \\
1.25 \\
1.50 \\
1.60\end{array}$ & $\begin{array}{l}2.65 \\
3.00 \\
3.00 \\
3.33 \\
2.30\end{array}$ & $\begin{array}{l}0.50 \\
0.64 \\
0.66 \\
0.94 \\
1.04\end{array}$ & $\begin{array}{l}\text { n.d. } \\
\text { n.d. } \\
\text { n.d. } \\
\text { n.d. } \\
\text { n.d. }\end{array}$ & $\begin{array}{l}3.75 \\
4.43 \\
4.50 \\
5.38 \\
5.00\end{array}$ & $\begin{array}{r}1900 \\
1550 \\
1850 \\
3550 \\
790\end{array}$ & $\begin{array}{l}105 \\
140 \\
139 \\
138 \\
121\end{array}$ & $\begin{array}{l}44 \\
56 \\
54 \\
69 \\
47\end{array}$ & $\begin{array}{l}39 \\
53 \\
25 \\
90 \\
32\end{array}$ & $\begin{array}{r}122 \\
93 \\
103 \\
95 \\
127\end{array}$ & $\begin{array}{r}359 \\
267 \\
236 \\
89 \\
122\end{array}$ & $\begin{array}{l}18 \\
23 \\
18 \\
34 \\
32\end{array}$ & $\begin{array}{l}15 \\
27 \\
24 \\
58 \\
66\end{array}$ & $\begin{array}{l}47 \\
33 \\
29 \\
49 \\
47\end{array}$ & $\begin{array}{r}89 \\
105 \\
89 \\
137 \\
100\end{array}$ \\
\hline $\begin{array}{l}55-1,10 \\
55-2,2 \\
56-3,10 \\
56-5,54 \\
57-4,76\end{array}$ & $\begin{array}{l}55.35 \\
56.75 \\
81.50 \\
58.00 \\
30.45\end{array}$ & $\begin{array}{r}19.74 \\
19.31 \\
5.48 \\
17.10 \\
10.27\end{array}$ & $\begin{array}{r}0.75 \\
0.88 \\
0.70 \\
1.30 \\
24.84\end{array}$ & $\begin{array}{l}2.98 \\
2.33 \\
0.59 \\
3.40 \\
1.54\end{array}$ & $\begin{array}{l}1.50 \\
1.58 \\
1.35 \\
1.15 \\
1.03\end{array}$ & $\begin{array}{l}2.55 \\
2.90 \\
1.15 \\
2.88 \\
1.35\end{array}$ & $\begin{array}{l}0.77 \\
0.73 \\
0.24 \\
0.40 \\
0.34\end{array}$ & $\begin{array}{l}\text { n.d. } \\
\text { n.d. } \\
0.21 \\
\text { n.d. } \\
\text { n.d. }\end{array}$ & $\begin{array}{l}4.93 \\
5.00 \\
1.10 \\
4.05 \\
2.24\end{array}$ & $\begin{array}{r}520 \\
490 \\
90 \\
320 \\
2890\end{array}$ & $\begin{array}{l}127 \\
116 \\
523 \\
159 \\
164\end{array}$ & $\begin{array}{r}56 \\
46 \\
9 \\
39 \\
18\end{array}$ & $\begin{array}{l}57 \\
57 \\
31 \\
98 \\
64\end{array}$ & $\begin{array}{r}106 \\
92 \\
77 \\
106 \\
37\end{array}$ & $\begin{array}{l}137 \\
185 \\
202 \\
148 \\
680\end{array}$ & $\begin{array}{r}23 \\
23 \\
0 \\
29 \\
29\end{array}$ & $\begin{array}{r}60 \\
48 \\
231 \\
63 \\
27\end{array}$ & $\begin{array}{l}51 \\
37 \\
30 \\
88 \\
35\end{array}$ & $\begin{array}{l}84 \\
92 \\
76 \\
84 \\
47\end{array}$ \\
\hline $\begin{array}{l}57-4,96 \\
59-3,33 \\
62-5,103 \\
63-3,45 \\
65-4,87\end{array}$ & $\begin{array}{l}41.40 \\
40.90 \\
53.90 \\
59.00 \\
35.80\end{array}$ & $\begin{array}{r}13.60 \\
12.19 \\
17.19 \\
17.76 \\
9.16\end{array}$ & $\begin{array}{l}23.65 \\
23.82 \\
12.42 \\
10.60 \\
29.77\end{array}$ & $\begin{array}{l}1.96 \\
2.98 \\
3.32 \\
3.15 \\
1.82\end{array}$ & $\begin{array}{l}1.15 \\
1.13 \\
1.30 \\
1.45 \\
0.93\end{array}$ & $\begin{array}{l}1.95 \\
1.95 \\
2.78 \\
2.78 \\
1.66\end{array}$ & $\begin{array}{l}0.43 \\
0.44 \\
0.50 \\
0.55 \\
0.35\end{array}$ & $\begin{array}{l}\text { n.d. } \\
\text { n.d. } \\
\text { n.d. } \\
\text { n.d. } \\
\text { n.d. }\end{array}$ & $\begin{array}{l}3.00 \\
3.25 \\
4.03 \\
4.25 \\
2.25\end{array}$ & $\begin{array}{r}1255 \\
1820 \\
830 \\
675 \\
4750\end{array}$ & $\begin{array}{l}160 \\
173 \\
166 \\
158 \\
140\end{array}$ & $\begin{array}{l}23 \\
20 \\
32 \\
33 \\
19\end{array}$ & $\begin{array}{l}46 \\
38 \\
84 \\
95 \\
46\end{array}$ & $\begin{array}{r}65 \\
67 \\
105 \\
109 \\
72\end{array}$ & $\begin{array}{l}373 \\
376 \\
119 \\
110 \\
384\end{array}$ & $\begin{array}{l}22 \\
17 \\
24 \\
27 \\
12\end{array}$ & $\begin{array}{r}60 \\
37 \\
64 \\
112 \\
36\end{array}$ & $\begin{array}{l}31 \\
34 \\
19 \\
24 \\
10\end{array}$ & $\begin{array}{r}78 \\
68 \\
105 \\
100 \\
63\end{array}$ \\
\hline $\begin{array}{l}66-2,17 \\
67-2,40 \\
71-2,120 \\
73-3,20 \\
74-2,28\end{array}$ & $\begin{array}{l}58.40 \\
39.20 \\
58.85 \\
39.20 \\
54.70\end{array}$ & $\begin{array}{l}17.48 \\
12.94 \\
17.38 \\
10.30 \\
15.68\end{array}$ & $\begin{array}{r}1.30 \\
16.17 \\
0.85 \\
26.34 \\
3.22\end{array}$ & $\begin{array}{l}3.27 \\
2.32 \\
3.27 \\
1.81 \\
3.98\end{array}$ & $\begin{array}{l}1.33 \\
1.05 \\
1.23 \\
1.00 \\
1.10\end{array}$ & $\begin{array}{l}2.58 \\
1.95 \\
2.85 \\
1.84 \\
2.80\end{array}$ & $\begin{array}{l}0.50 \\
0.39 \\
0.55 \\
0.29 \\
0.53\end{array}$ & $\begin{array}{l}\text { n.d. } \\
\text { n.d. } \\
\text { n.d. } \\
\text { n.d. } \\
\text { n.d. }\end{array}$ & $\begin{array}{l}4.66 \\
3.21 \\
4.56 \\
2.38 \\
4.40\end{array}$ & $\begin{array}{r}1350 \\
4875 \\
710 \\
4400 \\
2975\end{array}$ & $\begin{array}{l}157 \\
207 \\
154 \\
231 \\
246\end{array}$ & $\begin{array}{l}27 \\
25 \\
26 \\
33 \\
35\end{array}$ & $\begin{array}{r}84 \\
130 \\
95 \\
32 \\
157\end{array}$ & $\begin{array}{r}137 \\
78 \\
176 \\
64 \\
108\end{array}$ & $\begin{array}{r}113 \\
381 \\
78 \\
305 \\
110\end{array}$ & $\begin{array}{r}20 \\
17 \\
19 \\
8 \\
27\end{array}$ & $\begin{array}{l}56 \\
39 \\
68 \\
60 \\
69\end{array}$ & $\begin{array}{l}37 \\
32 \\
27 \\
22 \\
32\end{array}$ & $\begin{array}{r}113 \\
63 \\
121 \\
47 \\
142\end{array}$ \\
\hline $\begin{array}{l}75-3,67 \\
76-2,51 \\
82-3,48 \\
84-2,32 \\
85-4,87\end{array}$ & $\begin{array}{l}54.90 \\
56.90 \\
57.70 \\
50.60 \\
48.70\end{array}$ & $\begin{array}{l}14.64 \\
15.59 \\
19.74 \\
17.85 \\
15.59\end{array}$ & $\begin{array}{r}3.96 \\
11.68 \\
0.71 \\
5.18 \\
18.29\end{array}$ & $\begin{array}{l}3.98 \\
3.44 \\
2.63 \\
1.99 \\
1.69\end{array}$ & $\begin{array}{l}0.95 \\
1.23 \\
1.23 \\
1.50 \\
1.10\end{array}$ & $\begin{array}{l}2.65 \\
2.71 \\
3.45 \\
2.83 \\
2.65\end{array}$ & $\begin{array}{l}0.50 \\
0.55 \\
0.45 \\
0.50 \\
0.55\end{array}$ & $\begin{array}{l}\text { n.d. } \\
\text { n.d. } \\
\text { n.d. } \\
\text { n.d. } \\
\text { n.d. }\end{array}$ & $\begin{array}{l}4.27 \\
3.75 \\
4.24 \\
3.75 \\
3.50\end{array}$ & $\begin{array}{r}2775 \\
760 \\
440 \\
7625 \\
665\end{array}$ & $\begin{array}{l}143 \\
177 \\
188 \\
273 \\
177\end{array}$ & $\begin{array}{l}28 \\
32 \\
48 \\
54 \\
41\end{array}$ & $\begin{array}{r}61 \\
71 \\
71 \\
257 \\
40\end{array}$ & $\begin{array}{r}129 \\
78 \\
163 \\
145 \\
89\end{array}$ & $\begin{array}{r}119 \\
99 \\
76 \\
186 \\
208\end{array}$ & $\begin{array}{l}13 \\
17 \\
19 \\
28 \\
12\end{array}$ & $\begin{array}{l}69 \\
56 \\
80 \\
49 \\
33\end{array}$ & $\begin{array}{l}36 \\
17 \\
29 \\
49 \\
27\end{array}$ & $\begin{array}{r}105 \\
95 \\
113 \\
137 \\
89\end{array}$ \\
\hline $\begin{array}{l}92-3,68 \\
93-6,12^{\mathrm{a}} \\
93-6,12^{\mathrm{a}} \\
95-4,50 \\
98: 2,34^{\mathrm{a}}\end{array}$ & $\begin{array}{l}59.35 \\
56.95 \\
16.20 \\
57.90 \\
56.20\end{array}$ & $\begin{array}{r}19.84 \\
20.03 \\
3.50 \\
19.65 \\
18.89\end{array}$ & $\begin{array}{r}0.99 \\
1.04 \\
20.60 \\
0.93 \\
2.42\end{array}$ & $\begin{array}{l}2.20 \\
2.36 \\
1.51 \\
2.40 \\
2.16\end{array}$ & $\begin{array}{l}1.05 \\
1.23 \\
0.48 \\
1.23 \\
1.20\end{array}$ & $\begin{array}{l}2.58 \\
3.15 \\
0.60 \\
3.20 \\
3.00\end{array}$ & $\begin{array}{l}0.60 \\
0.58 \\
0.14 \\
0.60 \\
0.59\end{array}$ & $\begin{array}{l}\text { n.d. } \\
\text { n.d. } \\
1.58 \\
\text { n.d. } \\
\text { n.d. }\end{array}$ & $\begin{array}{l}4.31 \\
4.48 \\
4.20 \\
4.60 \\
3.96\end{array}$ & $\begin{array}{l}390 \\
460 \\
17.46^{\mathrm{b}} \\
530 \\
2180\end{array}$ & $\begin{array}{l}252 \\
230 \\
110 \\
230 \\
180\end{array}$ & $\begin{array}{l}61 \\
53 \\
19 \\
57 \\
58\end{array}$ & $\begin{array}{r}118 \\
100 \\
32 \\
190 \\
87\end{array}$ & $\begin{array}{r}158 \\
160 \\
63 \\
150 \\
150\end{array}$ & $\begin{array}{r}92 \\
87 \\
210 \\
94 \\
130\end{array}$ & $\begin{array}{r}23 \\
26 \\
0 \\
34 \\
18\end{array}$ & $\begin{array}{r}81 \\
62 \\
5 \\
66 \\
85\end{array}$ & $\begin{array}{l}36 \\
37 \\
22 \\
36 \\
35\end{array}$ & $\begin{array}{r}134 \\
134 \\
24 \\
152 \\
116\end{array}$ \\
\hline $\begin{array}{l}98-2,34 \\
99-2,74 \\
100-5,59 \\
101-4,38 \\
104-2,57\end{array}$ & $\begin{array}{r}8.60 \\
63.30 \\
36.10 \\
58.25 \\
41.45\end{array}$ & $\begin{array}{r}2.36 \\
18.80 \\
9.16 \\
17.40 \\
13.64\end{array}$ & $\begin{array}{r}23.02 \\
0.97 \\
16.83 \\
0.71 \\
16.10\end{array}$ & $\begin{array}{l}1.55 \\
2.32 \\
1.53 \\
2.09 \\
1.65\end{array}$ & $\begin{array}{l}0.45 \\
1.20 \\
0.75 \\
1.29 \\
1.05\end{array}$ & $\begin{array}{l}0.39 \\
2.95 \\
1.48 \\
2.63 \\
1.81\end{array}$ & $\begin{array}{l}0.09 \\
0.60 \\
0.29 \\
0.54 \\
0.43\end{array}$ & $\begin{array}{l}\text { n.d. } \\
\text { n.d.. } \\
\text { n.d. } \\
\text { n.d. } \\
\text { n.d. }\end{array}$ & $\begin{array}{l}4.05 \\
4.43 \\
3.43 \\
4.86 \\
3.80\end{array}$ & $\begin{array}{l}19.75^{\mathrm{b}} \\
750 \\
9.25^{\mathrm{b}} \\
740 \\
620\end{array}$ & $\begin{array}{r}230 \\
290 \\
400 \\
150 \\
84\end{array}$ & $\begin{array}{l}14 \\
52 \\
35 \\
42 \\
34\end{array}$ & $\begin{array}{r}38 \\
220 \\
170 \\
92 \\
50\end{array}$ & $\begin{array}{r}32 \\
150 \\
57 \\
130 \\
88\end{array}$ & $\begin{array}{r}260 \\
96 \\
150 \\
110 \\
260\end{array}$ & $\begin{array}{r}6 \\
44 \\
34 \\
26 \\
21\end{array}$ & $\begin{array}{r}6 \\
130 \\
67 \\
91 \\
100\end{array}$ & $\begin{array}{l}32 \\
41 \\
27 \\
37 \\
32\end{array}$ & $\begin{array}{r}0 \\
127 \\
84 \\
120 \\
100\end{array}$ \\
\hline $\begin{array}{l}106-5,56 \\
107-2,78 \\
108-6,119 \\
111-1,74 \\
116-1,91^{a}\end{array}$ & $\begin{array}{l}46.95 \\
56.75 \\
50.45 \\
57.50 \\
58.80\end{array}$ & $\begin{array}{l}13.85 \\
16.11 \\
15.33 \\
18.57 \\
15.92\end{array}$ & $\begin{array}{r}12.25 \\
9.45 \\
8.47 \\
0.62 \\
3.43\end{array}$ & $\begin{array}{l}1.67 \\
1.67 \\
1.97 \\
2.29 \\
2.44\end{array}$ & $\begin{array}{l}1.14 \\
1.06 \\
0.80 \\
1.31 \\
1.50\end{array}$ & $\begin{array}{l}2.00 \\
2.31 \\
2.28 \\
2.81 \\
2.38\end{array}$ & $\begin{array}{l}0.44 \\
0.49 \\
0.49 \\
0.65 \\
0.45\end{array}$ & $\begin{array}{l}\text { n.d. } \\
\text { n.d. } \\
\text { n.d. } \\
\text { n.d. } \\
\text { n.d. }\end{array}$ & $\begin{array}{l}4.45 \\
3.34 \\
4.15 \\
4.56 \\
3.39\end{array}$ & $\begin{array}{r}520 \\
1720 \\
520 \\
230 \\
720\end{array}$ & $\begin{array}{l}100 \\
180 \\
140 \\
170 \\
150\end{array}$ & $\begin{array}{l}31 \\
60 \\
31 \\
53 \\
40\end{array}$ & $\begin{array}{l}63 \\
40 \\
47 \\
48 \\
56\end{array}$ & $\begin{array}{r}97 \\
102 \\
105 \\
120 \\
97\end{array}$ & $\begin{array}{r}200 \\
211 \\
200 \\
96 \\
170\end{array}$ & $\begin{array}{r}22 \\
18 \\
16 \\
6 \\
5\end{array}$ & $\begin{array}{r}104 \\
66 \\
56 \\
110 \\
82\end{array}$ & $\begin{array}{l}24 \\
37 \\
37 \\
34 \\
24\end{array}$ & $\begin{array}{r}84 \\
116 \\
87 \\
140 \\
95\end{array}$ \\
\hline $\begin{array}{l}116-1,91^{3} \\
117-4,52 \\
117-4,76 \\
118-4,77 \\
118-6,44\end{array}$ & $\begin{array}{l}31.95 \\
68.55 \\
66.95 \\
57.85 \\
23.80\end{array}$ & $\begin{array}{r}8.35 \\
10.69 \\
11.10 \\
18.18 \\
7.89\end{array}$ & $\begin{array}{r}26.25 \\
3.62 \\
3.14 \\
0.95 \\
15.32\end{array}$ & $\begin{array}{l}1.63 \\
0.78 \\
1.26 \\
2.32 \\
3.73\end{array}$ & $\begin{array}{l}0.85 \\
1.63 \\
1.45 \\
1.33 \\
0.68\end{array}$ & $\begin{array}{l}1.35 \\
2.38 \\
2.13 \\
3.30 \\
1.33\end{array}$ & $\begin{array}{l}0.23 \\
0.43 \\
0.50 \\
0.58 \\
0.29\end{array}$ & $\begin{array}{l}\text { n.d. } \\
\text { n.d. } \\
\text { n.d. } \\
\text { n.d. } \\
\text { n.d. }\end{array}$ & $\begin{array}{c}1.80 \\
2.43 \\
2.39 \\
4.45 \\
18.00^{\mathrm{b}}\end{array}$ & $\begin{array}{l}1800 \\
240 \\
210 \\
300 \\
1.46^{b}\end{array}$ & $\begin{array}{l}130 \\
290 \\
160 \\
170 \\
190\end{array}$ & $\begin{array}{l}23 \\
42 \\
38 \\
77 \\
26\end{array}$ & $\begin{array}{l}37 \\
51 \\
42 \\
51 \\
25\end{array}$ & $\begin{array}{r}67 \\
67 \\
81 \\
110 \\
51\end{array}$ & $\begin{array}{l}260 \\
440 \\
320 \\
120 \\
110\end{array}$ & $\begin{array}{r}5 \\
42 \\
6 \\
17 \\
7\end{array}$ & $\begin{array}{r}53 \\
84 \\
119 \\
107 \\
13\end{array}$ & $\begin{array}{l}29 \\
32 \\
29 \\
43 \\
23\end{array}$ & $\begin{array}{r}53 \\
39 \\
63 \\
105 \\
32\end{array}$ \\
\hline $\begin{array}{l}119-3,60 \\
123-1,40 \\
126-3,78 \\
127-3,34 \\
130-5,29\end{array}$ & $\begin{array}{l}57.45 \\
47.00 \\
53.20 \\
52.60 \\
55.15\end{array}$ & $\begin{array}{l}18.77 \\
15.62 \\
17.69 \\
19.36 \\
19.06\end{array}$ & $\begin{array}{r}1.47 \\
8.61 \\
3.94 \\
10.14 \\
1.12\end{array}$ & $\begin{array}{l}1.68 \\
1.67 \\
1.66 \\
1.71 \\
1.68\end{array}$ & $\begin{array}{l}1.05 \\
1.00 \\
1.18 \\
1.18 \\
1.13\end{array}$ & $\begin{array}{l}3.10 \\
2.65 \\
3.30 \\
2.31 \\
3.25\end{array}$ & $\begin{array}{l}0.55 \\
0.51 \\
0.56 \\
0.60 \\
0.57\end{array}$ & $\begin{array}{l}\text { n.d. } \\
\text { n.d. } \\
\text { n.d. } \\
\text { n.d. } \\
\text { n.d. }\end{array}$ & $\begin{array}{l}3.48 \\
4.02 \\
3.70 \\
3.48 \\
3.63\end{array}$ & $\begin{array}{l}500 \\
930 \\
450 \\
300 \\
150\end{array}$ & $\begin{array}{l}190 \\
220 \\
180 \\
210 \\
220\end{array}$ & $\begin{array}{l}71 \\
68 \\
75 \\
89 \\
81\end{array}$ & $\begin{array}{l}71 \\
41 \\
47 \\
38 \\
56\end{array}$ & $\begin{array}{r}120 \\
98 \\
105 \\
100 \\
120\end{array}$ & $\begin{array}{r}140 \\
300 \\
170 \\
85 \\
110\end{array}$ & $\begin{array}{r}22 \\
9 \\
15 \\
13 \\
16\end{array}$ & $\begin{array}{l}23 \\
21 \\
19 \\
27 \\
20\end{array}$ & $\begin{array}{l}37 \\
47 \\
45 \\
24 \\
41\end{array}$ & $\begin{array}{l}130 \\
100 \\
105 \\
120 \\
120\end{array}$ \\
\hline $\begin{array}{l}131-1,9 \\
132-2,38 \\
134-4,45 \\
138-2,30\end{array}$ & $\begin{array}{r}19.85 \\
4.80 \\
5.20 \\
22.05\end{array}$ & $\begin{array}{l}6.49 \\
1.23 \\
1.28 \\
6.91\end{array}$ & $\begin{array}{l}36.73 \\
53.50 \\
52.00 \\
33.51\end{array}$ & $\begin{array}{l}1.34 \\
0.60 \\
0.61 \\
1.28\end{array}$ & $\begin{array}{l}1.35 \\
0.20 \\
0.18 \\
0.58\end{array}$ & $\begin{array}{l}1.25 \\
0.30 \\
0.30 \\
1.28\end{array}$ & $\begin{array}{l}0.23 \\
0.06 \\
0.06 \\
0.19\end{array}$ & $\begin{array}{l}\text { n.d. } \\
\text { n.d. } \\
\text { n.d. } \\
\text { n.d. }\end{array}$ & $\begin{array}{l}1.61 \\
0.53 \\
0.62 \\
1.80\end{array}$ & $\begin{array}{r}1210 \\
870 \\
930 \\
750\end{array}$ & $\begin{array}{l}130 \\
120 \\
150 \\
110\end{array}$ & $\begin{array}{r}21 \\
4 \\
5 \\
18\end{array}$ & $\begin{array}{l}24 \\
14 \\
17 \\
28\end{array}$ & $\begin{array}{l}61 \\
19 \\
25 \\
27\end{array}$ & $\begin{array}{l}560 \\
280 \\
330 \\
440\end{array}$ & $\begin{array}{r}7 \\
11 \\
12 \\
14\end{array}$ & $\begin{array}{r}85 \\
8 \\
12 \\
46\end{array}$ & $\begin{array}{r}110 \\
32 \\
31 \\
79\end{array}$ & $\begin{array}{l}58 \\
16 \\
11 \\
62\end{array}$ \\
\hline
\end{tabular}

a Duplicate analyses because of sediment heterogeneity. 
significant positive correlation of $\mathrm{Mg}$ with $\mathrm{Na}, \mathrm{Co}$, and elements associated with silicates; (2) an important increase of $\mathrm{Mn}$ which remains high up to the lower Oligocene; and (3) the disappearance of the positive correlation between $\mathrm{SiO}_{2}$ and $\mathrm{Li}$, with $\mathrm{Li}$ remaining bound to the $\mathrm{Fe}-\mathrm{Ti}-\mathrm{Al}$ group.

\section{Zone 6: Cores 398D-72 through 56; Middle Albian to Middle Cenomanian}

Claystones and mudstones of the upper part of Subunit 4A have a clay fraction characterized by the presence of abundant and well-crystallized smectite associated with small amounts of illite, kaolinite, and palygorskite with the same facies as in Zone 7. Mn is bound to $\mathrm{CaO}(+0.64)^{6}$. Only a few variations are noticed within this zone.

Near the top of Core 398D-56, however, just below the reddish Santonian sediments, the last Cenomanian deposits show a particular mineralogy. In the clay fraction, illite and chlorite are abundant and accompanied by mixed-layer clays, quartz, and amphiboles. Clinoptilolite is very abundant and generally present in vugs, mostly inside somewhat dissolved tests of foraminifers and radiolarians. SEM studies show prisms and blades of clinoptilolite associated with cristobalite with its characteristic lepispheroidal facies (Plate 1, Figures 5 and 6; Plate 2, Figure 1).

\section{Zone 5: Cores 398D-55 through 51; Lower Santonian}

This zone includes the barren mudstones and the brown to reddish claystones of the base of lithologic Sub-unit 3B. Ca and calcite disappear in the whole rock, while $\mathrm{Zn}, \mathrm{Cu}$ (perhaps bound to heavy minerals), $\mathrm{Fe}$, and $\mathrm{Ti}$ increase. The clays are composed of abundant smectite associated with attapulgite and kaolinite. Illite is rare; sepiolite appears for the first time, but is also rare. The unusually high percentages of $\mathrm{Zn}$ and $\mathrm{Cu}$ might be related to locally identified heavy minerals.

\section{Zone 4: Cores 398D-50 through 41; Santonian, Campanian, Maestrichtian}

Zone 4 includes dark red mudstones of lithologic Sub-units $3 \mathrm{~B}$ and $3 \mathrm{~A}$. Its top corresponds to the Cretaceous/Tertiary boundary. Calcite reappears and the $\mathrm{Sr} / \mathrm{CaO}$ ratio has normal values. In the clay fraction, fresh micas-illites are abundant, and smectites are relatively rare (Plate 2, Figure 2).

Besides illites, the following minerals were noticed: chlorite; kaolinite; irregular mixed-layer illite-smectite, illite-vermiculite, and chlorite-smectite; quartz; feldspars; and sometimes amphiboles and goethite. Attapulgite is rare and sometimes absent, except in Core 398D-44 where the clay fraction contains abundant smectite, attapulgite, and traces of sepiolite.

\footnotetext{
${ }^{6}$ Correlation coefficient value.
}

\section{Zone 3: Cores 398D-40 through 33; Paleocene, Lower Eocene}

The brown calcareous mudstones of this zone are characterized by a high $\mathrm{Mg}$ content related to the abundance of fibrous minerals in the clay fraction. This second fibrous clay minerals episode includes sepiolite, in addition to attapulgite. Compared with those of the $\mathrm{Al}$ bian, the fibers have different characteristics; they are very long, thin, sometimes curved, well-individualized, and electron refractive. Associated small idiomorphic rhombic minerals are probably dolomite crystals (Plate 2, Figures 3 and 4). Moreover, SEM studies show that attapulgite grows at the edge of phyllites, probably smectite (Plate 2, Figure 5). Chlorite and kaolinite, as well as mixed-layer clays are absent. Associated nonclay minerals are traces of feldspars, quartz (sometimes abundant at the bottom of this zone), and clinoptilolite. When studied in detail, the following clay mineral sequence can be observed: Core $398 \mathrm{D}-40$ is dominated by attapulgite; Core 398D-39 to Section 398D-35-5, by smectite; Sections 398D-35-2 to 398D-33-4, by sepiolite; then attapulgite again dominates in Core 398D-33.

This zone is marked by the largest amounts of $\mathrm{Mg}$ and by the fluctuation of the $\mathrm{MgO} / \mathrm{K}_{2} \mathrm{O}$ ratio. In addition, a high covariant factor groups $\mathrm{MgO}, \mathrm{Na}_{2} \mathrm{O}, \mathrm{SiO}_{2}$, $\mathrm{Al}_{2} \mathrm{O}_{3}$, and Co. $\mathrm{Ca}$ joins the above elements in Core 398D-34, where calcite and sepiolite are abundant. Core 398D-34 also shows a further increase of the $\mathrm{MgO} / \mathrm{K}_{2} \mathrm{O}$ ratio. Additionally, Cores 398D-40 and 38 include the last phosphatic intervals at this site.

\section{Zone 2: Cores 398D-34 through 20(?); Middle and Upper Eocene}

Zone 2 includes all except the base of lithologic Unit 2 . The top of Zone 2 is difficult to define with precision because Cores 398D-18 and 19 were not studied. The zone is rich in smectite, which typifies Cretaceous sediments. Small quantities of short, straight, lath-shaped attapulgite and sepiolite fibers are present. Quartz and feldspars, especially alkaline feldspars, were noticed in the clay fraction. Illite increased a little from the upper Eocene upwards (above Core 398D-23), and chlorite is present throughout this zone.

Calcite is scarce throughout this zone. Mn, abundant as deep as the Albian sediments (first episode with fibrous clay minerals), decreases markedly and nearly disappears after a temporary occurrence in Cores 398D-23 and 20. $\mathrm{Na}_{2} \mathrm{O}$ and $\mathrm{MgO}$ lose all significant correlation with other chemical elements.

Zone 1: Cores 398D-17(?) through 2, 398B-1, 398A-2 and 1, and 398-4 through 1; Oligocene to Pleistocene

This zone includes most of lithologic Unit 1 and differs from Zone 2 by a large increase in calcite (mean values of $14 \%$ in Core 398D-20, 54\% in Core 398D-17). Calcite decreases only near the top, in Pleistocene deposits $(25-30 \%)$. 
Another characteristic of this zone is the permanence of correlation between different elements. The $\mathrm{Sr}$ shows high values (up to $1850 \mathrm{ppm}$ ) which correlates strongly with calcite between the bottom of Zone 1 and Core 398A-2. Clay minerals exhibit an evolution marked by illite, chlorite, and kaolinite increasing at the expense of smectite and fibrous clays, then by the reoccurrence of mixed-layers illite-smectite and chlorite-smectite, quartz, and feldspars. Sepiolite is missing in Zone 1, attapulgite very rare (Plate 2, Figure 6).

The arrival of more abundant typical detrital clay minerals (illite, chlorite) upward in Zone 1 is noticed, especially in the upper Oligocene, the upper Miocene. In Pleistocene sediments, these minerals are largely dominant.

\section{DISCUSSION}

Before considering the significance of mineralogical and geochemical assemblages in the nine zones identified at Site 398, it is useful to explain briefly the main possible origins of the various constituents (see Millot, 1964; Biscaye, 1965; Krauskopf, 1967; Griffin et al., 1968; Paquet, 1969; Chamley, 1971; Latouche, 1971a; Wise et al., 1972; Lomova, 1975; Stonecipher, 1976; Weaver and Beck, 1977).

Illite, chlorite, quartz, amphiboles, as well as calcicsodic and alkali feldspars are present in crystalline and metamorphic rocks. Their occurrence can result from the rejuvenation of nearby mountains by tectonism, or of the appearance of streams draining old shield areas. Among chemical characteristics, $\mathrm{Li}$ is often associated with this mineral group (particularly with illite and alkali feldspars).

Kaolinite occurs particularly in soils of hot and humid climates by weathering in a well-drained environment. Its increasing amount in the sea corresponds to either a more hydrolyzing climate or to recurring erosion of continental basins. Kaolinite is associated with $\mathrm{Zn}$.

Smectite can have various origins: degradation in well-drained soils in mild climates, neoformation in badly drained soils in hot climates with contrasting seasons, sedimentary formation in a basic chemically confined environment, or by aerial or submarine transformation of volcanic materials. The abundant smectites at Site 398 usually are marked by the presence of $\mathrm{Al}, \mathrm{Fe}, \mathrm{Ti}, \mathrm{Cr}, \mathrm{V}$, and $\mathrm{Li}$.

Fibrous clays (attapulgite $=$ palygorskite, sepiolite) are due to either submarine evolution of hyd othermal and/or volcanic products, or sedimentary growth in a basic, chemically confined environment. The same is true for zeolites of the clinoptilolite family and for cristobalite. Sepiolite is associated with Co and perhaps $\mathrm{Ni}$. The whole set of fibrous clays determines a statistical covariation of $\mathrm{Mg}$ with the silicate phase but they are antagonist to $\mathrm{Li}$. The inverse relationship of elements in the couple $\mathrm{MgO}-\mathrm{Li}$ seems to characterize the presence of fibrous clays.

Irregular mixed-layers (illite-vermiculite, illitesmectite, chlorite-vermiculite, chlorite-smectite) are generally good criteria of the continental alteration of primary clay minerals. They are mainly developed in middle latitudes.

Calcite is basically of biological marine origin (planktonic and benthic). It is marked by $\mathrm{Sr}$ and also can have a partially continental origin. Pyrite, siderite, rhodochosite, and gypsum were formed in the sediments during their deposition or shortly thereafter. They correspond to reducing or locally oxidizing conditions, and can determine important increases of $\mathrm{Mn}$ and/or $\mathrm{Fe}$.

These different primary sources are not necessarily responsible for the deposit of the minerals found at Site 398. Different origins are possible, particularly from the erosion of outcropping sediments or from the reworking of unconsolidated sediments deposited on the continental shelf or slope.

Let us now examine how these differing origins can affect the Cretaceous and Tertiary history near Site 398. We will endeavor to present the most probable hypothesis for each zone, and introduce other possible hypotheses. A reasonable concept of the genetic mechanisms that are responsible for the mineral and geochemical associations which have taken place since the Early Cretaceous can only be obtained by comparing data from many Atlantic sites similar to Site 398.

\section{Hauterivian, Lower Barremian-Zone 9}

The oldest Cretaceous deposits penetrated (Cores 398D-138 to 131), as well as the most important part of Cretaceous and Paleogene sediments are marked by very abundant and highly crystallized smectite. The origin of this mineral, which occurs over a long time span and with changing lithology, does not favor in-situ formation. The sedimentary components in this interval show the importance of detrital source (either transported by turbidities or in hemipelagic form) and the lack of authigenic minerals (Site Report; de Graciansky; Bourbon, both, this volume). Cristobalite and zeolites exist locally above Zone 9; however, their presence does not correspond with an increase in smectite. Minerals of volcanic origin also occur very rarely. That is why, for most of the smectites at Site 398, we do not favor an in-situ genesis by alteration of volcanic material or by chemical sedimentation. Smectite is chiefly a terrigenous mineral, which is corroborated by its affinity with $\mathrm{Ti}, \mathrm{Cr}$, and $\mathrm{V}$, those elements being typically linked with a detrital origin. Furthermore, rare earths, as determined by C. Courtois (University of Paris, personal communication), are characteristic of a continental origin, without any marine or volcanic contribution. Their composition (sum of all elements $=100$ to $120 \mathrm{ppm}$ from 20 samples studied), which shows a low impoverishment in heavy components ( $\mathrm{Tb}, \mathrm{Yb}, \mathrm{Lu})$, is close to that of typical continental shales.

In Hauterivian sediments, as well as in most later deposits, the smectite probably originates from gently sloping, poorly drained continental soils which are common during ante-alpine periods. The abundant formation of pedogenic, well-crystallized smectites indicates a tropical to hot Mediterranean climate, rather arid with strong seasonality (Paquet, 1969). The brief wet season permits upward hydrolysis and ionic mobilization; the 
marked dry season is responsible for the ions becoming trapped in the low-lying ground soils ("vertisols") and for their formation into smectites. This essentially pedologic origin is substantiated by the predominant alumino-ferric nature of the chemical association (Trauth et al., 1967). It is conceivable that part of the smectites also result from the erosion of basic chemical sediments (Millot, 1964). The extreme rarity of kaolinite favors a climate drier in Zone 9 that in younger sediments, i.e., either the formation of kaolinite restricted on the continent, or the dry climate did not favor fluvial transport.

Part of the mineral assemblage could be inherited from pre-Hauterivian, smectite-rich sedimentary rocks. However, this is highly improbable because the Lower Cretaceous of DSDP Site 397 (south of the Canaries) and of DSDP Site 416 (off Morocco) is especially rich in illite and chlorite (Tithonian to lower Hauterivian; Chamley and Giroud d'Argoud, this volume, Part 1; and unpublished data). It is possible, however, that illite from Hauterivian deposits at Site 398 might be inherited from older sedimentary rocks. The same possibility exists for traces of fibrous clays in Zone 9: they may be derived from the erosion of early Mesozoic rocks (Lucas, 1962; Weaver and Beck, 1977).

The abundance of calcite (up to $95 \% \mathrm{Co}_{3} \mathrm{Ca}$ ) and consequently of $\mathrm{Ca}$ in some intervals contrary to the relative rareness of $\mathrm{Sr}$, is a problem because these two elements are usually in association with one another in marine sediments. A deficit of $\mathrm{Sr}$ occurs in many carbonaceous sediments deposited in epicontinental seas (Debrabant, 1970). It might result from the diagenesis of pelagic carbonaceous sediments, the recrystallization causing the loss of a part of the $\mathrm{Sr}$ (Odum, 1975). This change could appear during the transformation of aragonite into calcite (Wolf et al., 1967), which occurs in intermediate water depths. Such a phenomenon is in agreement with an epicontinental environment for the Hauterivian limestones. The fairly high Mn content suggests another possibility; namely, the precipitation of carbonates in an environment marked by low salinity (Sr impoverishment), low $\mathrm{ph}$ (Mn enrichment), and low temperature (Zeller and Wray, 1956).

\section{Barremian, Aptian, Lower and Middle Albian-Zone 8}

The mineralogical and geochemical Zone 8, about 500 meters thick (Cores 398D-130 to 89), corresponds to a complex, heterogeneous sedimentation, comprised of the irregular alternation of local and allochthonous deposits with changing sequence (Bourbon; de Graciansky; Sigal; all this volume). This instability in the sedimentation accounts for the following major characteristics: changing mineralogical associations; statistical independence of most chemical elements; intense fluctuations of the $\mathrm{Sr} / \mathrm{CaO}$ ratio; and the presence of localized intervals with particular minerals.

Nevertheless, the constant presence of smectite in the argillaceous phase as well as the continuity of geochemical covariant criteria (excepting the Barremian) indicates a unique origin within this sedimentary se- quence. This is in agreement with the idea of an undersea talus fan in a zone of subsidence, proposed by de Graciansky (this volume) from his study of facies and sedimentary sequences at Site 398.

Smectite, whose characteristics are the same as those of Zone 9, has an identical prevailing origin, i.e., an inheritance from poorly drained continental soils formed during a hot climate with contrasting seasons. Continental moisture might be slightly more important than during the Hauterivian, if we judge from the increase in kaolinite and from the disappearance of fragile fibrous clays. But that change could also reflect a tectonic rejuvenation of continental relief (see Groupe Galice, this volume; Rehault and Mauffret, this volume), perhaps related to the last phases of oceanic rifting (Sibuet and Ryan, this volume). In Zone 9, the reworking of older sediments contributes to the detrital supply which is testified to by the presence of Tithonian Calpionelleds in some intervals (Cores 398D-124 and 106, Clansayesian; Sigal, this volume). This could also indicate a minor tectonic event on the surrounding landmasses.

The temporary decreases of smectite in Zone 8, leading to the identification of Sub-zone 8A (Barremian and lower Albian), can be explained in two ways. We can imagine a change in the nature of mineral and biogenic detrital supplies. Thus, a certain chemical relationship exists between organic carbon and the $\mathrm{Al}-\mathrm{K}-\mathrm{Ti}$ phase, which shows the detrital and continental origin of the organic material. The cause could be partly climatic or partly tectonic rejuvenation, as assumed above. A tectonic cause is reasonable during the Barremian, where a strong geophysical break occurs between Cores 398D-131 and 130 (see Site Report, this volume). This suggests a continental reaction to oceanic rifting (Sibuet et al., this volume). During the late Aptian and early Albian (Cores 398D-101/100), the second mineralogical decrease of smectite could be related to the first stages of sea-floor spreading (Sibuet and Ryan, this volume; Montadert et al., in press) which would be responsible for increasing subsidence of the continental margin and, therefore, continental erosion.

Another explanation exists for the decreases of smectite observed in Sub-zone 8A. The systematic relationship which appears between the organic carbon concentration and the mineralogical composition of the argillaceous phase, a relationship which begins at the top of the Hauterivian interval, recalls one which exists in Pliocene-Quaternary sapropelic intervals of the eastern Mediterranean (Chamley, 1971; Sigal et al., 1978). There, when organic carbon increases, smectite decreases in favor of irregular mixed-layer clays, illite, and perhaps chlorite. The nature of the mineralogical change observed in black sediment intervals seems to indicate a modification of in-situ organic acid effects. Smectite is formed as small particles with high ion exchange capability. This mineral (more than others) absorbs organic matter, which is able to cause silicate framework alterations (Huang and Keller, 1971). Mixed-layer clays with transitory degradation characters are produced, whereas strong minerals such 
as illite show a relative increase. The degradation proposed here is moderate and, relative to the five alteration stages in organic environment proposed for the Mediterranean (Sigal et al., 1978), at most it reaches stage two at Site 398. It is a common suite of the heterogeneous and disturbed features of sedimentation, plus a moderate abundance of organic material (organic $\mathrm{C}<3 \%$ ). A more distinctive alteration in a more confined environment would produce a disappearance of mixed-layers and a degradation of illite. According to the hypothesis of an in-situ clay degradation, the alteration seems stronger in the second organic zone (Cores 398D-101 to 92) than in the first one (Cores 398D-134 to 119). This is in agreement with a less disturbed and more reduced depositional environment which would be propitious for chemical reactions. A recurrence in the concentration of organic material, higher in the hole ( top of Core 398D-56) is also noteworthy; it could have produced an effect similar to the one observed here, on the abundance and crystallinity of smectite.

The richer intervals of organic material often are also the layers of higher concentrations of $\mathrm{Fe}$ and $\mathrm{Mn}$, elements located in nearby Ca-rich zones. These concentrations are marked by rhodochrosite or siderite. Formation of such minerals presupposes high concentrations of dissolved carbonates, a deficit in sulfur (Krauskopf, 1967), and a 6.5 to $7.0 \mathrm{ph}$ (Garrels and Christ, 1967). The mechanism for such an evolution in sediments is in agreement with the above-mentioned hypothesis for the partial degradation of clay-minerals. This hypothesis presumes an early weak diagenesis in an anoxic environment which causes the hydrolysis of certain clays, the dissolution of the carbonates and the leaching of $\mathrm{Ca}$ and of such metalliferous elements as $\mathrm{Fe}$ and $\mathrm{Mn}$. This results in their diffusion into sediments and precipitation of metalliferous carbonates adjacent to organic intervals.

Other intervals in Zone 8 are characterized by the presence of zeolites and cristobalite, the origin of which can be autochthonous or allochthonous. Zeolites of the clinoptilolite family are usually not found in association with volcanic glass or abundant smectite. They correspond to minima in smectite and to a marked increase in detrital micas. Moreover, sandy quartz is abundant which results in a high $\mathrm{SiO}_{2} / \mathrm{Al}_{2} \mathrm{O}_{3}$ ratio (6.0 to 6.5, against 2.4 to 3.0 for zeolites in the absence of free $\mathrm{SiO}_{2}$; Stonecipher, 1976; Couture, 1977). These features favor zeolites being inherited from the continental margin by slope currents. The currents can result from a syn-sedimentary tectogenesis, connected with the subsidence of the basin (de Graciansky, this volume). Clinoptilolite and perhaps cristobalite could have been formed in marginal basins. This problem will be discussed in the Zone 6 discussion, for which more data are available. We must underline that average or low metallization in zeolite intervals excludes the possibility of a volcanic origin for those minerals.

\section{Middle Albian-Zone 7}

A strong break occurs in the mineralogical and geochemical composition of Core 398D-78, which is not related to a lithological change (within a succession of dark shales with various reworked intercalated sediments). Attapulgite, which had been rare to absent, suddenly becomes abundant and thus continues until Core 398D-73. The large content of this fragile fibrous clay and its association with $\mathrm{Mg}$ shows the lack of noticeable degradation and the organic environment and minor degree of evolution of Albian black shales. The "black shale" facies, extending a considerable distance in the Atlantic Ocean during the Cretaceous and presenting a high carbonaceous content consequently has diversified characteristics chemical in time and space (Ryan and Cita, 1977).

The origin of attapulgite seems easily established. The mineral occurs in a fine-grained detrital sedimentary sequence, deposited in an open ocean environment (de Graciansky, this volume) when detrital supplies from the continental margin increased (Bourbon, this volume). Attapulgite appears without perceptible change in conditions of deposition. Moreover, it occurs in short fibers independent of other minerals and quite similar to Pliocene-Quaternary attapulgites inherited from the African area (Chamley and Millot, 1975; Chamley et al., 1977; Weaver and Beck, 1977). This points to a detrital origin for the Albian attapulgite at Site 398. A major physiographic change could have provided outcrops of old attapulgite bearing rocks (Triassic?), the erosion of which would have supplied the marine environment. But no structural evidence exists to support such a hypothesis. Attapulgite probably was formed in Iberia during the Albian. The poor preservation of the mineral (Plate 1, Figures 3 and 4) suggests a long transport history, leading to the fragmentation and dissociation of its fibers. This would imply a distant origin. The abundance, dispersion, and morphological facies do not support the possibility of volcanic or hydrothermal formation as sometimes occurs (Bonatti and Joensuu, 1968; Hoffert et al., 1975). The mineral is likely to have formed under basic chemical conditions, perhaps locally at the same time as smectite (Millot et al., 1957; Millot, 1964; Trauth, 1974; Weaver and Beck, 1977), in a marginal marine basin fairly distant from Site 398. Attapulgite was then reworked and transported under the same unstable detrital sedimentary regime which characterizes lithologic Unit 4 (Site Report, this volume). The chemical formation of attapulgite implies a warm and hydrolyzing continental climate, as well as a low-relief topography propitious to ionic trapping. Note the absence of sepiolite associated with attapulgite. This could arise from a less-hydrolyzing climate or from less favorable transport conditions for mineral preservation than during the Paleocene-Eocene ages (see Zone 3).

The occurrence of attapulgite coincides with an increase in $\mathrm{Mg}$. This correspondence poses a problem because in the overlying series the presence of $\mathrm{Mn}$ is independent from fibrous clays; the chemical element remains abundant, whereas the mineralogical composition shows large quantitative changes. The statistical study does not show any direct relationship between Mn and detrital minerals. The factor analysis shows that a 
part of the Mn is connected to a complex metallic phase including $\mathrm{Ni}, \mathrm{Zn}$, and $\mathrm{Co}$. This metalliferous increase might be linked only fortuitously with the arrival of fibrous clays and actually might be due to a physicalchemical change, such as the supply of carbonaceous detrital elements. In this regard, a significant relationship is observed between $\mathrm{Mn}$ and $\mathrm{CaO}$ in mineralogical Zone 7 , then subsequently disappears.

Note that the smectite supply in Zone 7 has the same chemical associations as before, marked by $\mathrm{Fe}, \mathrm{Al}, \mathrm{Ti}$, and $\mathrm{Li} . \mathrm{Li}$ is inversely related to fibrous clays, and consequently loses its significant relationship with silica, while $\mathrm{Mg}$ becomes covariant $\left\{\mathrm{R}\left(\mathrm{SiO}_{2}, \mathrm{MgO}\right)=0.70\right\}$. The chemical stability of smectite suggests that the part of the mineral which could have been formed in the same conditions as attapulgite is very weak, which confirms the above-mentioned observations.

\section{Upper Albian, Cenomanian-Zone 6}

From a mineralogical and geochemical point of view, the upper sequence of "black shales" (Cores 398D-70 to 56) is the most homogeneous at Site 398. It is marked by high contents of well-crystallized smectite, which demonstrates the weakness of interactions between organic and mineral matter, and reflects a low organic evolution (Deroo et al., this volume). The nature of smectite indicates a warm humid climate, propitious to soil development in low-lying and poorly drained areas. The primary detrital mineral (illite) is rare, and kaolinite is very rare, which suggests either the minor influence of erosion and transport from upstream continental zones or a drier climatic average than before. Attapulgite occurs as short fibers in all samples, and was probably derived from the erosion of Albian rocks (Zone 7).

The uppermost part of Zone 6 (Core 398D-56), which corresponds locally to a gap, causes a genetic problem because of the occurrence of zeolite and cristobalite at the transition to the overlying Santonian beige muds. These minerals could have two origins, each of them being backed by strong arguments. The first proposed origin is by autochthonous crystallization during slow deposition, thereby permitting chemical changes. Clinoptilolite is often present in tablets and prisms, which are in an excellent state of preservation despite their fragile nature. It is associated with cristobalite, whose fragile "lepisphere" structure (Plate 1, Figure 6; Plate 2 , Figure 1) does not seem able to endure significant transport. The genesis of the minerals could benefit by local hydrothermal or volcanic contributions. This explanation is supported by the proximity of Vigo Seamount and the existence of a marked instability in the Iberian peninsula during the Upper Cretaceous (Boillot and Capdevila, 1977). Such an origin by autochthonous precipitation in a volcanic environment is described by numerous authors, in the Upper Cretaceous and Paleogene sediments of DSDP drill sites (Deffeyes, 1959; von Rad and Rösch, 1972; Wise and Kelts, 1972; Venkatarathnam and Biscaye, 1973; Aubry and Pomerol, 1975; Flörke et al., 1976; Pomerol, 1976; von
Rad et al., in press). Nevertheless, a volcanic influence is not essential; an in-situ formation of silicate minerals is possible in a normal marine interstitial environment, especially in conjunction with biogenic silica derived from planktonic shells (diatoms, radiolarians: see Millot, 1964; Mitsui and Taguchi, 1977). This possibility is supported by the relative independence of the occurrence of zeolite and cristobalite.

The second origin presupposes a detrital input of clinoptilolite and/or cristobalite; these minerals originate through chemical sedimentation in Atlantic continental margin areas, without volcanic or interstitial intervention. Arguments for this origin are based on the high detrital character of the sedimentation: gradedbedding in the strata, and an abundance of quartz, illite, and chlorite. The excellent preservation of the zeolite and cristobalite minerals could have resulted from their formation inside calcareous tests (chiefly foraminifers), as suggested by microscope studies. Such a location would have protected the fragile crystals from abrasion during transport. Free zeolites are often less wellpreserved than those enclosed in tests. The absence of transition metals ( $\mathrm{Mn}, \mathrm{Ni}, \mathrm{Co}$ ) does not support the idea of a volcanic origin. The relative abundance of $\mathrm{Zn}$ and $\mathrm{Cu}$ can easily be explained by the noticeable occurrence of heavy minerals in the detrital-rich intervals. These observations support the interpretation of Sommer (1972), Wise et al. (1972), Leclaire et al. (1973), Leclaire (1974), Chumakov and Shumenko (1977), and Weaver and Beck (1977) about the crystallization of these minerals in shallow water in a confined chemical environment. The minerals would then have been reworked, fostered by the instability of the subsiding African margin.

Both possible origins are supported by strong arguments and it is difficult to favor one over the other at present. The discussion does lead to an absence of certainty about the relationship between clinoptilolite, cristobalite, and volcanism/hydrothermalism. This opinion is shared by Brown et al. (1969) and Stonecipher (1976). It is possible that both origins envisaged here were contributors or that the genesis of both groups of minerals is partly independent.

\section{Santonian - Zone 5}

The beige-reddish muds of Cores 398D-55 to 51 are characterized by the lack of $\mathrm{Ca}$ and an enrichment in $\mathrm{Fe}$ and $\mathrm{Ti}$. If the disappearance of $\mathrm{Ca}$ was affected by a rise in the CCD, this rise should also have affected the underlying strata, whose chemical composition is similar to the present one (specifically, Core 398D-82 and 71). The beige color, locally associated with the presence of goethite and subamorphous iron oxides, could result partly from an incipient deep oceanic circulation system (e.g., see Laughton, Berggren et al., 1972; Le Pichon et al., in press). Nevertheless, one cannot observe in Zone 5 any increase in the amount of minerals coming from high latitudes (micas, chlorites). Intense continental erosion triggered by proper climatic or tectonic conditions could lead to similar effects. The 
occurrence of noticeable quantities of attapulgite, in addition to smectite and the appearance of small amounts of sepiolite, suggest a warmer climate than before. An increase in kaolinite indicates increasing humidity. Both these observations indicate that the mineralogical and geochemical changes occurring in the lower Santonian are due to climate modifications rather than to changes in physiography in the source area or oceanic currents.

\section{Santonian, Campanian, Maestrichtian - Zone 4}

The mineralogical changes identified at the base of the Santonian deposits (attapulgite increasing, and the appearance of sepiolite, which seem to indicate a climatic change (see Zone 3 ), are abruptly interrupted by a strong increase in primary minerals and kaolinite (Cores 398D-51 to 41). The sudden appearance of illite and chlorite associated with quartz, feldspars, and amphiboles, is quite unexpected in a mineralogical cycle characterized by smectite and fibrous clays, which disappear over more than 100 meters in depth.

The explanation for this important mineralogical break must first be sought in a major tectonic event on the landmasses near Site 398. In this regard, the period extending from the late Cretaceous to the Eocene corresponds to the time of uplift of the Pyrenean mountains, as a probable consequence of the subduction and collision of Europe and Iberia (Choukroune, 1973; Boillot and Capdevila, 1977). The uplift of the Pyrenean chains, accompanied by the renewal of relief surrounding areas, fits in with the marked mineralogical change observed in Maestrichtian deposits. The presence of kaolinite agrees with this explanation, as positive tectonic movements favor the accentuation of erosion in the most upstream parts of river basins in which this mineral is preferentially formed in soils. Chemically, the reappearance of $\mathrm{Ca}$ in calcitic form could be due to the erosion of old limestone strata. Note that similar effects (red-beige sediments, and an increase in primary minerals and kaolinite content) would also occur with a marine regression during the Maestrichtian, an event comparable or consecutive to the one beginning during the mid-Cenomanian in many parts of the North Atlantic and adjacent land masses (Hart and Tarling, 1974).

Another possibility is that a rapid widening of the North Atlantic Ocean during Maestrichtian time may have led to the formation of large meridinal currents which transported northern latitude minerals to middle latitudes. The parallel increase of kaolinite and primary minerals does not necessarily contradict this possibility; kaolinite occurs in various Mesozoic rocks at high latitudes (Darby, 1975).

Two arguments support this hypothesis. First, tectonism does not justify a strong decrease in the supply of primary minerals during the Paleocene, even though uplift was fairly continuous and even increased toward Eocene time. Our hypothesis is better suited with an initial phase during which a distant source was contributing (illite, chlorite, etc.), then a decrease in this supply because of an equilibration with the local supply once a new erosional regime was established. Secondly, it is difficult to conceive the same tectonic event affecting the Iberian Peninsula and northeastern Biscay Bay, whereas a modification in the general oceanic circulation could affect both areas.

If the hydrodynamic hypothesis were confirmed, one could consider the detrital minerals (especially the smaller-sized clay minerals) as useful markers in the dating of major stages of oceanic opening. In this case, one would propose a middle Santonian-CampanianMaestrichtian age for a possible circulation event, perhaps related to the spacing between Greenland and Canada (Laughton, Berggren et al., 1972; Berggren and Hollister, 1974), and perhaps occurring in two stages (Figure 1).

It is not presently possible to make allowances for each hypothesis at Site 398. The uniformity of the geochemical parameters during the Maestrichtian, especially that of the $\mathrm{Sr} / \mathrm{CaO}$ ratio, suggests a single continuing origin for detrital minerals and carbonaceous fractions. A single event could be responsible for the unique mineralogical assemblage characterizing Zone 5. This problem will have to be considered from a broader view of the geochemical and mineralogical data obtained on numerous Upper Cretaceous sediments drilled in the North Atlantic.

\section{Paleocene, Lower Eocene - Zone 3}

The second zone marked by fibrous clays (Cores 398D-40 to 33) differs greatly from Albian zones. Sepiolite occurs in addition to attapulgite, and the fibers are long and well preserved. Attapulgite seems to be derived from the transformation of smectite, whose peripheral blades it often prolongs in a delicate hair-like texture. Such a phenomenon has been described by Trauth (1974) and Weaver and Beck (1977). All these mineralogical and morphological characteristics evoke an autochthonous formation for the fibrous clays.

In spite of these observations, all the lithological data point to a detrital character for the sedimentation: slumped muds, turbidites and numerous reworkings, open-sea sediments (Site Report, this volume). It is obvious, therefore, that the Paleocene and Eocene fibrous clays are detrital, like their Albian counterparts. This is in agreement with the data obtained off Morocco (DSDP Leg 41), where a sepiolite-rich and attapulgiterich turbidite interval more than 300 meters thick was recognized in the Eocene (Lancelot, Seibold, et al., 1977). Similarly, Latouche (1971b) discovered Paleocene clay breccias with attapulgite on the Cantabria Seamount (Bay of Biscay). This conclusion corresponds to Weaver and Beck's (1977) opinion concerning the detrital origin of most marine palygorskites, except for local hydrothermal formations. A recent example is the occurrence of great amounts of Holocene detrital sepiolite on the continental margin of northern Africa (Froget and Chamley, 1977). All these observations indicate the caution necessary in deciphering the autochthonous or allochthonous origin of minerals, including the most fragile ones such as sepiolite and attapulgite. In the present example, the only definite factor is that the transport distance of fibrous clays was 
short and that it was performed in turbiditic masses, where individual particles were shielded.

If Paleogene attapulgite and sepiolite are detrital, their formation and origin are still to be determined. Several authors think that these minerals were formed in an oceanic volcanic or hydrothermal environment (e.g., Bowles et al., 1971; von Rad and Rösch, 1972, Lomova, 1975). This interpretation, which locally finds justification in typical hydrothermal deposits (Hathaway and Sachs, 1965; Bonatti and Joensuu, 1968), does not agree very well with their widespread geographical and temporal occurrence in marine beds, with the almost general independence of these clays from hydrothermal or volcanic indices, and with their narrow tolerance of the climatic environment (Weaver and Beck, 1977). Indeed, most marine attapulgites and sepiolites are developed in marginal basins by chemical sedimentation in basic (often carbonated) environments, in subtropical to equatorial climates. This was the old interpretation of Millot et al. (1957) and Millot (1964), who found confirmation in Paleocene lacustrine or oceanic margin environments, in hot and wet climates (Prévôt et al., 1971; Triat and Trauth, 1972; Trauth, 1974; see also bibliography in Millot, 1964; Chamley, 1971; Weaver and Beck, 1977). The genesis of fibrous clays in confined basins occurs in a complex mineralogical and geochemical sequence. It is often in association with other minerals (i.e., clinoptilolite and cristobalite) and, in the presence of organic matter, can be replaced laterally by a phosphate sequence or by hydrocarbonated sediments (Millot, 1964; Prévôt et al., 1971; Lucas and Prévôt, 1975; Weaver and Beck, 1977).

The relative spacing of samples at Site 398 and the clastic character of the sedimentation does not allow us to state if the mineralogical series from the Paleocene to lower Eocene agrees with the geochemical sequence (attapulgite, smectite, and sepiolite). The only pertinent evidence is the existence during this period of a hot and wet climate, as well as relatively isolated ocean margins where minerals were deposited through erosion and seaward transport. Their final resting place was influenced by subsidence and reworking by oceanic currents. These observations, corroborated by the correlation between sepiolite and $\mathrm{Mg}$ and carbonate, indicate chemical sedimentation. Changing $\mathrm{MgO} / \mathrm{K}_{2} \mathrm{O}$ and $\mathrm{Sr} / \mathrm{CaO}$ ratios account for the mineralogical complexity as evidenced by the development of geochemical sequences and their irregular removal. The occasional occurrence of phosphate marks the proximity and diversity of confined continental margin basin. The strong chemical tie between $\mathrm{MgO}, \mathrm{Co}$, and $\mathrm{Na}_{2} \mathrm{O}$ is an indication of the relative abundance of fibrous clays throughout this zone.

\section{Middle and Upper Eocene - Zone 2}

Beginning with Core 398D-32, the amount of fibrous clay decreases rapidly while illite increases. Smectite remains predominant as it has been since the Lower Cretaceous, and still indicates a warm climate although cooler than during the Paleocene and lower Eocene. This moderate climatic change, also reflected by a discrete increase in illite and by traces of chlorite mixed- layer minerals, agrees with the incipient cooling indicated by planktonic foraminiferal assemblages (Iaccarino and Premoli-Silva, this volume). Fibrous clay abundance progressively decreases, and sepiolite disappears completely in the lower Oligocene (Core 398D-20). Locally, these minerals were still generated during this period, even until the upper Miocene (Weaver and Beck, 1977), in confined and lacustrine basins (Millot, 1964). An example of this genesis of fibrous clays exists in Galicia on the Iberian Peninsula (Lucas et al., 1963). At Site 398, the fibrous clays seem to be chiefly inherited from older sedimentary rocks, which supplied them in decreasing amounts as new rocks and soils developed on the continent (see Chamley, 1975). Towards the top of Zone 2, the small increase in kaolinite might imply a more humid climate, or the last stage of major subsidence related to oceanic spreadings. Meanwhile, and for the first time since the Albian (Zone 7), the manganiferous flux decreases while the significant correlations of $\mathrm{Na}_{2} \mathrm{O}$ and $\mathrm{MgO}$ disappear.

\section{Oligocene to Pleistocene - Zone 1}

Successive changes occur from Core 398D-16 upwards, with most of these changes having the same signification. In spite of widely spaced samples in the upper part of the hole, these changes can be briefly discussed by referring to known post-upper Paleocene phenomena.

The increase in chloritic and illitic minerals during the Oligocene implies a cooling climate which continued cooling until Pleistocene time. An acceleration of the opening of the Atlantic Ocean may have had an additional effect, inducing a deep circulation not very different from today's. An important and abrupt increase in calcareous deposits substantiates this argument. Where $\mathrm{Ca}$ and $\mathrm{Sr}$ correlate, the calcareous deposits have a marine origin and their development agrees with a broad open ocean which permits pelagic and homogeneous sedimentation. These ideas agree with various recent views concerning: (a) the late opening of some North Atlantic areas, (b) a worldwide cooling period during the upper Oligocene, and (c) the age of initiation of the deep-sea circulation (Laughton, Berggren et al., 1972; Hayes, Frakes et al., 1975; Kennett, Houtz et al., 1975; Le Pichon et al., in press).

During the middle and upper Miocene, illite and chlorite increase, as does irregular mixed-layers of illitesmectite and chlorite-smectite, and quartz and feldspars. This is probably related to a worldwide cooling during Cenozoic time, contemporaneous with the formation of Antarctic island ice (Kennett and Brunner, 1973; Ryan et al., 1974; Hayes, Frakes, et al., 1975; Kennett, Houtz, et al., 1975). Subsequently, medium latitude areas were submitted to a moderately temperate climatic environment which continues to the present. On subaerially exposed soils, this cooling causes a decrease in chemical and an increase in mechanical weathering. This is why mineral products washed to the sea originate less from soil erosion than from moderately weathered rocks. This is also what accounts for the increase in primary clay minerals, slightly alterated to irregular mixed-layer minerals. As for kaolinite, which is 
equally well represented in Neogene sediments, it is probably derived from ancient soils which were eroded because of physiographical factors (rejuvenation) and/ or climatic ones (increase in pluvial conditions following more arid periods which generated smectite). One notes a temporary increase in all the minerals toward the end of the Miocene (Core 398D-3), which might indicate renewed erosion, possibly correlating with the "Messinian" regression, the results of which are important in the Mediterranean Sea (Clauzon, 1973; Ryan et al., 1974), on the Atlantic margin of Africa (DiesterHaass and Chamley, in press), and in austral seas (Kennett, 1967).

During Pleistocene (Cores 398-4 to 2) illite, chlorite, and irregular mixed-layer minerals constitute more than half of the clay fraction, while calcite and Ca decreases. These conditions are determined in medium latitudes by glacial cycles which favor the development of mechanical erosion processes. Furthermore, the development of cool deep-sea currents originating in high latitudes favors the dissolution of calcareous tests (e.g., Melguen and Thiede, 1975; Leclaire and Clocchiatti, 1976), as well as a rise of the lysocline.

\section{CONCLUSION}

Nine mineralogical and geochemical zones were recognized in the Cretaceous and Cenozoic section drilled at Site 398.

Specific associations between minerals and the chemical composition provided information about the origin and diagenesis of the sediments. Moreover, they provided data about hypotheses relating to regional geology, climatology, physiography, and global tectonics as follows:

1) Most minerals are detrital in origin and are inherited from diversified sources. This is true even for Cretaceous and Paleogene smectites which originated from soils covering the hinterland. Moreover, the habit of Albian attapulgite and of Paleocene attapulgite and sepiolite, all marked by Co, show that even these minerals are inherited from soils or continental margin basic basins.

Calcite sometimes has a detrital origin even in the Hauterivian where the low $\mathrm{Sr} / \mathrm{CaO}$ ratio indicates a diagenetic secondary evolution. There, the origin of the limestone can be on the shelf, from intermediate oceanic depths, or even from older sedimentary outcrops on land. Part of the clinoptilolite, perhaps part of the cristobalite, could also be inherited because these minerals are frequently associated with typical detrital minerals.

2) There is an absence of any significant diagenetic evolution which could be related to the depth of burial. Minerals such as smectite, attapulgite, sepiolite, and clinoptilolite, which disappear rapidly with increasing pressure and temperature are present almost to the bottom of the hole. Moreover, the variability of the clay minerals and associated trace elements as well as the evolution of the $\mathrm{Sr} / \mathrm{CaO}$ ratio reflects sedimentary events and shows that mineral recrystallization due to depth of burial has not started yet.

However, early diagenetic changes involving the formation of authigenic minerals were noticed in some intervals. These changes involve some of the clinoptilolite crystals and cristobalite lepispheres found in intergranular areas. The silica for their formation was provided by the dissolution of siliceous tests. These changes occur in the older rocks (Late Cretaceous). Another possible example of in-situ early diagenetic evolution is a moderate degradation of smectite in Lower Cretaceous organic-rich intervals, similar to the decay of clays in sapropelic environments. The geochemistry within such intervals favors the mobility of elements like $\mathrm{Ca}, \mathrm{Fe}, \mathrm{Mg}$, and $\mathrm{Mn}$. This accounts for local concentrations of secondary calcite, siderite, rhodocrosite, and dolomite associated with pyrite within reduced Aptian sediments.

Consequently, except for local environmental conditions, the detrital origin of most of the minerals reflects geological events affecting large areas.

3) Climate strongly influences the type of rock alteration on land and the nature of clay minerals formed in soils, which are subsequently eroded and transported to the ocean floor. The Cretaceous and Paleogene are characterized by the abundance of smectite accompanied by $\mathrm{Fe}, \mathrm{Ti}, \mathrm{Cr}, \mathrm{V}$, and partly $\mathrm{Li}$. Smectite is considered to be inherited chiefly from soils of hot arid climates, with strongly contrasting dry and wet seasons. From middle-late Oligocene to the present, the irregular increase in primary minerals, chiefly illite and chlorite, indicates a progressive world cooling, the main periods being Oligocene, late Miocene, and Pleistocene. In detail, the following climatic evolution can be deduced from the detrital clay assemblage:

Hauterivian and early Barremian, hot and dry.

Late Barremian and early Albian, possibly a little wetter.

Middle Albian, hot and wet.

Late Albian to Cenomanian, hot and fairly dry.

Santonian to early Eocene, becoming hotter and wetter with a maximum near the Paleocene/Eocene boundary.

Middle and late Eocene, hot and drier.

Oligocene to Pleistocene, progressively colder with numerous fluctuations and variable humidity.

4) Physiographic changes in the hinterland, coast, and sea floor also influence sedimentation. The formation of fibrous clays and smectites and the concentration of related elements $\left(\mathrm{Si}, \mathrm{Mg}\right.$, and $\left.\mathrm{P}_{2} \mathrm{O}_{5}\right)$ require continental margin basins having poor communication with the open sea. Albian, Paleocene, and Eocene fibrous clays in particular certainly formed under such conditions. Their presence in the open sea sediments then can be explained by coastal reworking, accompanied by irregular subsidence. Moreover, tectonic movements and erosion of sedimentary outcrops possibly explain the presence of at- 
tapulgite in the upper Albian-Cenomanian, attapulgite and sepiolite in the upper Eocene-Oligocene, and kaolinite in the Miocene-Pliocene-Pleistocene. Pyrenean movements may be responsible for the arrival of fresh minerals (illite, chlorite, quartz, feldspars, and amphiboles) during Santonian-Maestrichtian times.

5) At present, few relationships can be definitely demonstrated between the opening of the North Atlantic and the mineralogy of the sediments. Most sediment constituents such as smectite, attapulgite, and sepiolite seem to be inherited from the Iberian continent or shelf area. Weathered volcanic glass or rocks derived from the Mid-Atlantic Ridge do not contribute to the sedimentation of Site 398. Even zeolites and cristobalite which are often described as resulting from the submarine alteration of volcanic rocks or ash, were formed here either on the continental shelf or during early diagenesis in normal interstitial water. However, some increases of kaolinite and primary minerals could reflect the reactions of continental and continental margin areas to the late stages of oceanic rifting (middle Barremian) or to the beginning of active sea-floor spreading (uppermost Aptian).

The presence of less-degraded detrital minerals in the Late Cretaceous could be related to the separation of Greenland and Canada, which facilitated broad northsouth water circulation. Deep oceanic currents and associated North Atlantic minerals characterize Site 398 middle-upper Oligocene sediments.

\section{ACKNOWLEDGMENTS}

The mineralogical study carried out in Marseilles benefited by the financial support of CNEXO-FRANCE (Grant No. 76/5320) and by fruitful discussions with M. Arthur, C. Courtois, F. McCoy, J.-P. Herbin, W. B. F. Ryan, and J.-C. Sibuet. The geochemical studies in Lille received the financial support of CNEXO (Grant No. 76/5319) and the assistance of M. Meunier and B. Jouglet. Data obtained in the Institut de Géologie du Bassin d'Aquitaine (Bordeaux, C.N.R.S. Associate Laboratory No. 197) were supported by Action Thématique Programmée No. 2685 of C.N.R.S. France. The entire text and illustrations benefited from the reviews of P. C. de Graciansky, W. B. F. Ryan, and J.-C. Sibuet.

\section{REFERENCES}

Aubry, M.-P. and Pomerol, B., 1975. La pétrogenése des craies du Bassin de Paris est-elle une conséquence de l'expansion océanique?, C. R. Acad. Sci., Paris, sér. D, v. 280 , p. 2081-2084.

Berggren, W. A. and Hollister, C. D., 1974. Paleogeography, paleobiogeography and the history of circulation in the Atlantic region. In Hay, W. W. (Ed.), Studies in Paleooceanography, Soc. Econ. Petrol. Miner., Spec. Publ. 20, p. 126-186.

Biscaye, P. E., 1965. Mineralogy and sedimentation of recent deep-sea clay in the Atlantic ocean and adjacent seas and oceans, Geol. Soc. Am. Bull., v. 76, p. 803-832.
Boillot, G. and Capdevila, R., 1977. The Pyrenees: subduction and collision?, Earth Planet. Sci. Lett., v. 35, p. 151-160.

Bonatti, E. and Joensuu, O., 1968. Palygorskite from Atlantic deep-sea sediments, Am. Mineralogist, v. 53, p. 975-983.

Bowles, F. A., Angino, E. A., Hosterman, J. W., and Galle, O. K., 1971. Precipitation of deep-sea palygorskite and sepiolite, Earth Planet. Sci. Lett., v. 11, p. 324-332.

Brown, G., 1961. The X-ray identification and crystal structure of clay minerals, Miner. Soc., London.

Brown, G., Catt, J. A., and Weir, A. H., 1969. Zeolites of the clinoptilolite-heulandite type in sediments of southeast England, Miner. Mag., v. 37, p. 480-488.

Chamley, H., 1971. Recherches sur la sédimentation argileuse en Méditerranée, Sci. Géol. Mém., Strasbourg, no. 35. , 1975. Sédimentation argileuse en mer Tyrrhénienne au Plio-Pléistocène d'après l'étude du forage JOIDES 132, Bull. Groupe Franç. Argiles, v. 27, p. 97-137.

Chamley, H. and Millot, G., 1975. Observations sur la répartition et la génèse des attapulgites plio-quaternaires de Méditerranée, C. R. Acad. Sci., Paris, sér. D, v. 281, p. $1215-1218$.

Chamley, H., Diester-Haass, L., and Lange, H., 1977. Terrigenous material in East Atlantic sediment cores as an indicator of NW African climates, "Meteor" Forsch.Ergebnisse, v. 26, p. 44-59.

Choukroune, P., 1973. Phase tectonique d'âge variable dans les Pyrénnées: évolution du domaine plissé au cours du Tertiaire, C. R. Acad. Sci., Paris, sér. D, v. 276, p. 909-912.

Chumakov, I. S. and Shumenko, S. I., 1977. Heulandite authigène des dépôts pliocènes d'embouchure du Nil, $C . R$. Acad. Sci., Paris, sér. D, v. 284, p. 333-336.

Clauzon, G., 1973. The eustatic hypothesis and the prePliocene cutting of the Rhone valley. In Ryan, W.B.F., Hsü, K. J., et al., Initial Reports of the Deep Sea Drilling Project, v. 13: Washington (U.S. Government Printing Office), p. 1251-1256.

Couture, R. A., 1977. Composition and origin of palygorskiterich and montmorillonite-rich zeolite-containing sediments from the Pacific Ocean, Chem. Geol., v. 19, p. 83-90.

Darby, D. A., 1975. Kaolinite and other clay minerals in Arctic Ocean sediments, J. Sediment. Petrol., v. 45, p. 272 279.

Debrabant, P., 1970. Typologie géochimique des calcaires. Application à l'étude de l'origine des calcaires métamorphiques des massifs hercyniens français, Thèse, Sci. Nat., Lille.

Deffeyes, K. S., 1959. Zeolites in sedimentary rocks, J. Sediment. Petrol., v. 29, p. 602-609.

Diester-Haass, L. and Chamley, H., in press. Neogene paleoenvironment investigations off NW Africa (DSDP Leg 14) by means of a coarse and clay fraction analysis, J. Sediment. Petrol.

Flörke, O. W., Hollmann, R., von Rad, U., and Rösch, H., 1976. Intergrowth and twinning in opal-CT lepispheres, Contrib. Mineral. Petrol., v. 58, p. 235-242.

Froget, C. and Chamley, H., 1977. Présence de sépiolite dans les sédiments récents du golfe d'Arzew (Algérie), $C . R$. Acad. Sci., Paris, sér. D, v. 285, p. 307-310.

Garrels, R. M. and Christ, C. L., 1967. Equilibre des minéraux et de leurs solutions aqueuses: Paris (Gauthier-Villars Edit.).

Griffin, J. J., Windom, H., and Goldberg, E. D., 1968. The distribution of clay minerals in the world ocean, Deep-Sea Res., v. 15, p. 433-459. 
Hart, M. B. and Tarling, D. H., 1974. Cenomanian paleogeography of the North Atlantic and possible mid-Cenomanian eustatic movements and their implications, Palaeogeogr., Palaeoclimat., Palaeoecol., v. 15, p. 95-108.

Hathaway, J. C. and Sachs, P. L., 1965. Sepiolite and clinoptilolite from the mid-atlantic ridge, Am. Mineralogist, v. 50 , p. $852-867$.

Hayes, D. E., Frakes, L. A., et al., 1975. Initial Reports of the Deep Sea Drilling Project, v. 28: Washington (U.S. Government Printing Office).

Hoffert, M., Lalou, C., Brichet, E., Bonte, P., and Jehanno, C., 1975. Présence en Atlantique Nord de nodules de manganèse à noyaux d'attapulgite et de phillipsite authigènes, C. R. Acad. Sci., Paris, sér. D, v. 281, p. 231-233.

Huang, W. H. and Keller, W. D., 1971. Dissolution of clay minerals in dilute organic acids at room temperature. $\mathrm{Am}$. Mineralogist, v. 56, p. 1082-1095.

Kennett, J. P., 1967. Recognition and correlation of the Kapitean stage (Upper Miocene), New Zealand, New Zealand J. Geol. Geoph., v. 10, p. 1051-1063.

Kennett, J. P. and Brunner, C. A., 1973. Antarctic Late Cenozoic glaciation. Evidence for initiation of ice rafting and inferred increased bottom-water activity, Geol. Soc. $\mathrm{Am}$. Bull., v. 84 , p. $2043-2052$.

Kennett, J. P., Houtz, R. E., et al., 1975. Initial Reports of the Deep Sea Drilling Project, v. 29: Washington (U.S. Government Printing Office).

Krauskopf, K. B., 1967. Introduction to geochemistry: New York (McGraw Hill).

Lancelot, Y., Seibold, E., et al., 1977. Initial Reports of the Deep Sea Drilling Project, v. 41: Washington (U.S. Government Printing Office).

Latouche, C., 1971a. Les argiles des bassins alluvionnaires aquitains et des dépendances océaniques. Contribution à l'étude d'un environnment, Thèse, Sci. Nat., Bordeaux, $415 \mathrm{p}$.

1971b. Découverte d'attapulgite dans les sédiments carottés sur le dôme Cantabria (Golfe de Gascogne). Conséquences paléogéographiques, C. R. Acad. Sci., Paris, sér. D, v. 272 , p. 2064-2066.

Laughton, A. S., Berggren, W. A., et al., 1972. Initial Reports of the Deep Sea Drilling Project, v. 12: Washington (U.S. Government Printing Office).

Leclaire, L., 1974. Hypothèse sur l'origine des silicifications dans les grands bassins océaniques. Le rôle des climats hydrolysants, Bull. Soc. Géol. France, sér. 7, v. 16, p. 214-224.

Leclaire, L., Alcaydé, G., and Froehlich, F., 1973. La silicifation des craies: rôle des sphérules de cristobalite-tridymite observés dans les craies des bassins océaniques et dans celles du Bassin de Paris, C. R. Acad. Sci., Paris, sér. D, v. 277, p. 2121-2124.

Leclaire, L. and Clocchiatti, M., 1976. La dissolution des carbonates en milieu océanique. Son rôle dans la genèse des dépôts pélagiques pendant le Cénozoíque, Bull. Soc. Géol. France, sér. 7, v. 18, p. 1315-1335.

Le Pichon, X., Melguen, M., and Sibuet, J.-C., in press. A schematic model of the evolution of the South Atlantic. Joint Oceanogr. Assembly, Edinburg.

Lomova, O. S., 1975. Abyssal palygorskite clays of the Eastern Atlantic and their genetic relation to alkalic volcanism (from data of Legs 2 and 14 of the Glomar Challenger), Litol. i Polzn, Iskop., v. 4, p. 10-27.

Lucas, J., 1962. La transformation des minéraux argileux dans la sédimentation. Etudes sur les argiles du Trias, Mém. Serv. Carte Géol. Alsace-Lorraine, Strasbourg, no. 23.
Lucas, J. and Prévôt, L., 1975. Les marges continentales, pièges géochimiques; l'exemple de la marge atlantique de l'Afrique à la limite Crétacé-Tertiaire, Bull. Soc. Géol. France, sér. 7, v. 17, p. 496-501.

Lucas, J., Nonn, H., and Paquet, H., 1963. Présence de niveaux à sépiolite et attapulgite dans les sédiments tertiaires de Galice (Espagne), Bull. Serv. Carte Géol. AlsaceLorraine, Strasbourg, v. 16, p. 227-232.

Melguen, M. and Thiede, J., 1975. Influence des courants profonds au large du Brésil sur la distribution des faciès sédimentaires récents. Ninth Intern. Congr. Sedim., Nice, v. 8 , p. 51-55.

Millot, G., 1964. Géologie des Argiles: Paris, (Masson Edit.), p. 499.

Millot, G., Radier, H., and Bonifas, M., 1957. La sédimentation argileuse à attapulgite et montmorillonite, Bull. Soc. Géol. France, sér. 6, v. 7, p. 425-434.

Mitsui, K. and Taguchi, K., 1977. Silica mineral diagenesis in Neogene Tertiary shales in the Tempoka district, Hokkaido, Japan, J. Sediment. Petrol., v. 47, p. 158-167.

Montadert, L. C., Roberts, D. G., et al., in press. Rifting and subsidence on Passive Continental Margins in the North East Atlantic, Nature.

Odum, H. T., 1975. Biochemical deposition of strontium, Texas Univ., Inst. Mar. Sci., v. 4, p. 39-114.

Paquet, H., 1969. Evolution géochimique des minéraux argileux dans les altérations et les sols des climats méditerranéens et tropicaux à saisons contrastées, Mém. Serv. Carte Géol. Alsace-Lorraine, Strasbourg, no. 30.

Pinta, M., 1971. Spectrométrie d'absorption atomique. Masson (Edit.), Paris.

Pomerol, B., 1976. Géochimie des craies du cap d'Antifer (Haute-Normandie), Bull. Soc. Géol. France, sér. 7, v. 18, p. 1051-1060.

Prévôt, L., Boulo, A., and Lucas, J., 1971. Etude minéralogique de la série phosphatée des Ganntour (Maroc). Résultats préliminaires, Bull. Serv. Carte. Géol. AlsaceLorraine, Strasbourg, v. 24, p. 243-253.

Ryan, W.B.F. and Cita, M. B., 1977. Ignorance concerning episodes of ocean-wide stagnation, Mar. Geol., v. 23, p. 197-215.

Ryan, W.B.F., Cita, M. B., Rawson, D., Bruckle, L. H., and Saito, T., 1974. A paleomagnetic assignment of Neogene stage Boundaries and the development of isochronous datum planes between the Mediterranean, the Pacific and Indian oceans in order to investigate the response of the world ocean to the Mediterranean "salinity crisis," Riv. Ital. Paleontol., v. 80, p. 631-688.

Sigal, W., Chamley, H., Fabricius, F., Giroud d'Argoud, G., and Müller, J., 1978. Sedimentology and environmental conditions of sapropels. In Hsü, K. J., Montadert, L. C., et al., Initial Reports of the Deep Sea Drilling Project, v. 42, Part 1: Washington (U.S. Government Printing Office), p. 445-465.

Sommer, F., 1972. Néogenése de clinoptilolite dans l'Eocène et l'Oligocène de Casamance (Sénégal), Sci. Géol. Bull., Strasbourg, v. 25 , p. 251-258.

Stonecipher, S. H., 1976. Origin, distribution and diagenesis of phillipsite and clinoptilolite in deep-sea sediments, Chem. Geol., v. 17, p. 307-318.

Thorez, J., 1975. Phyllosilicates and clay minerals: Dison (Lelotte Edit.), p. 582.

Trauth, N., 1974. Argiles évaporitiques dans la sédimentation carbonatée tertiaire. Bassins de Paris, de Mormoiron et de 


\section{H. CHAMLEY ET AL.}

Salinelles (France); Jbel Ghassoul (Maroc), Thése, Sci. Nat., Strasbourg.

Triat, J. M. and Trauth, N., 1972. Evolution des minéraux argileux dans les sédiments paléogènes du bassin de Mormoiron (Vaucluse), Bull. Soc. Franç. Minér. Crist., v. 95, p. 482-494.

Venkatarathnam, K. and Biscaye, P. E., 1973. Deep-sea zeolites: variations in space and time in the sediments of the Indian Ocean, Mar. Geol., v. 15, p. M11-M17.

von Rad, U., Riech, V., and Rösch, H., 1977. Silica diagenesis in continental margin sediments off Northwest Africa. In Lancelot, Y., Seibold, E., et al., Initial Reports of the Deep Sea Drilling Project, v. 41: Washington (U.S. Government Printing Office), p. 879-906.

von Rad, U. and Rösch, H., 1972. Mineralogy and origin of clay minerals, silica and authigenic silicates in Leg 14 sediments. In Hayes, D. E., Pimm, A. C., et al., Initial Reports of the Deep Sea Drilling Project, v. 14: Washington (U.S. Government Printing Office), p. 727-751.

Weaver, C. E. and Beck, K. C., 1977. Miocene of the S.E. United States: a model for chemical sedimentation in a perimarine environment, Sediment. Geol., v. 17, p. 234.

Wise, S. W., Jr., Buie, B. F., and Weaver, F. M., 1972. Chemically precipitated cristobalite and the origin of chert, Ecolog. Geol. Helv., v. 65, p. 157-163.

Wise, S. W., Jr., Kelts, K. R., 1972. Inferred diagenetic history of a weakly silicified deep sea chalk, Gulf Coast Assoc. Geol. Soc. Trans., v. 22, p. 177-203.

Wolf, K. H., Chilingar, G. V., and Beales, F. W., 1967. Elemental composition of carbonate skeletons minerals and sediments, Carbonate rocks: New York (Elsevier).

Zeller, E. J. and Wray, J., 1956. Factors influencing precipitation of calcium carbonate, Am. Assoc. Petrol. Geol. Bull., v. 40 , p. $140-152$. 

PLATE 1

DSDP Site 398 Electron-Micrographs

Figure 1 Sample 398D-127-3, $34 \mathrm{~cm} .5000 \times$. Barremian. Large illite, small smectite with diffuse skirts.

Figure 2 Sample 398D-99-2, $74 \mathrm{~cm} .7200 \times$. Lower Albian. Smectite in great fleecy particles. Illite with sharp edges.

Figure 3 Sample 398D-75-3, $67 \mathrm{~cm}$. 4000×. Albian. Attapulgite (palygorskite) in short and straight fibers. Minor smectite and kaolinite.

Figure 4 Sample 398D-75-3, $67 \mathrm{~cm} .22,500 \times$. Minor smectite and kaolinite.

Figure 5 Sample 398D-57-5, $74 \mathrm{~cm} .2500 \times$. Cenomanian. Prisms of clinoptilolite in a matrix of coccoliths and clays.

Figure 6 Sample 398D-56-1, $72 \mathrm{~cm} .3000 \times$. Cenomanian. Lepispheres of cristobalite and clinoptilolite, inside a foraminiferal test. 
PLATE 1
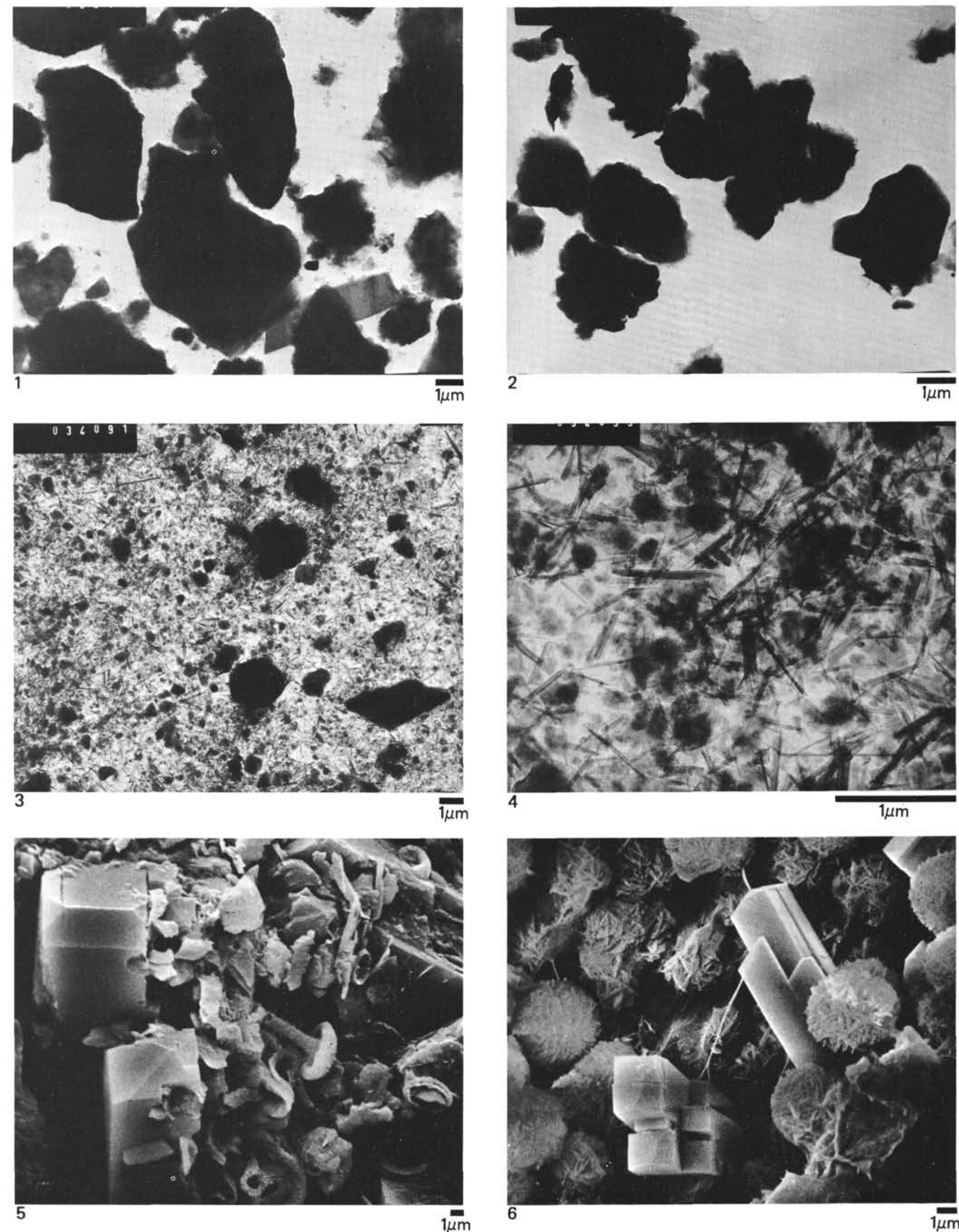
PLATE 2

\section{DSDP Site 398 Electron-Micrographs}

Figure 1 Sample 398D-56-1, $72 \mathrm{~cm} .5000 \times$. Cenomanian. Lepispheres of cristobalite and clinoptilolite, inside a foraminiferal test.

Figure 2 Sample 398D-41-1, $118 \mathrm{~cm} .22,500 \times$. Maestrichtian. Large illite particles, kaolinite hexagons, fleecy smectite, attapulgite fibers.

Figure 3 Sample 398D-34-1, $38 \mathrm{~cm} .11,500 \times$. Lower Eocene. Long flexuous sepiolite fibers, independent from other minerals. Square or lozenges of dolomite?

Figure 4 Sample 398D-34-1, $38 \mathrm{~cm} .22,500 \times$. Lower Eocene. Long flexuous sepiolite fibers, independent from other minerals. Square or lozenges of dolomite?

Figure 5 Sample 398D-40-4, $80 \mathrm{~cm}$. 10,000 $\times$. Paleocene. Attapulgite (palygorskite) growing on smectite sheets.

Figure 6 Sample 398D-2-1, $98 \mathrm{~cm} .22,500 \times$. Pleistocene. Illite and chlorite with shaped contours. Hexagonal kaolinite. Fairly rare attapulgite fibers. Fleecy smectite. 


\section{PLATE 2}
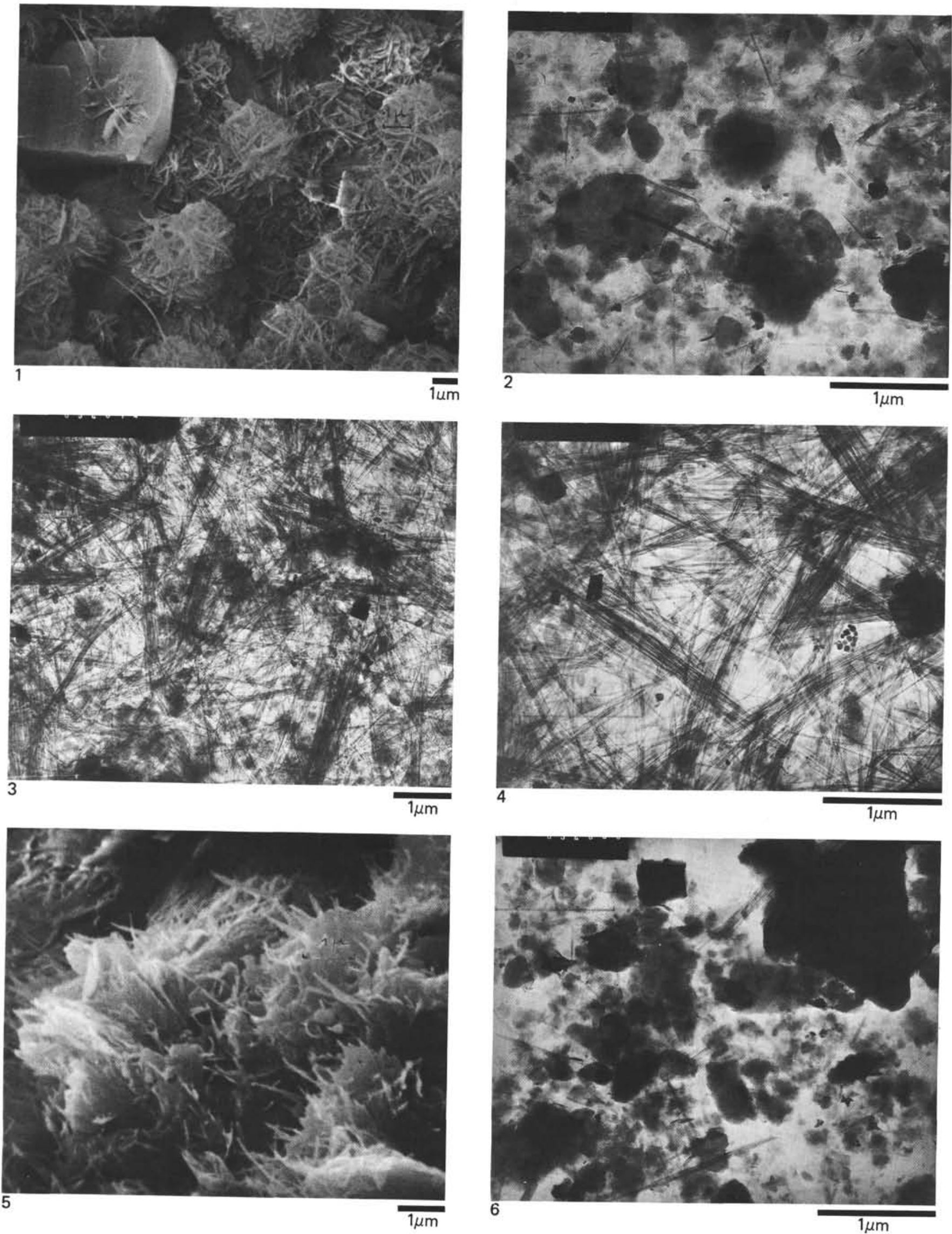